Polymer Journal, Vol. 8, No. 1, pp 96-113 (1976)

\title{
Theory of Liquid-Liquid Interface of Polymer Systems
}

\author{
Takuhei NosE \\ Department of Polymer Chemistry, Tokyo Institute \\ of Technology, Meguro-ku, Tokyo 152, Japan.
}

(Received August 4, 1975)

\begin{abstract}
A theory for the interfacial tension and interface thickness of demixed polymer mixtures and polymer solutions is presented on the basis of the theory of Cahn and Hilliard, taking into account the change in the dimension of polymer coil at the interface. The following results are obtained from the theory: (1) Near critical temperature $T_{\mathrm{c}}$ in a polymer mixture with the same chain length $m$ (a symmetrical system), interfaical tension $\sigma$ increases with $m$ in the $\frac{1}{2}$ power of $m$, whereas in a polymer solution, $\sigma$ decreases with $m$ in the- $\frac{1}{4}$ power, compared at the same reduced temperature $T / T_{c}$. On the other hand, the thickness of the interface near $T_{\mathrm{c}}$ increases with increasing $m$, proportional to the unperturbed chain dimension, i.e., to $m^{1 / 2}$, in a symmetrical polymer system, and to the $\frac{1}{4}$ power in a polymer solution. (2) In high polymer systems, a first order transition from a diffuse interface to a sharp interface is predicted. The transition temperature reduced by $T_{\mathrm{c}}$ increase with increasing $m$. The thickness of the interface at a low temperature does not greatly depend on $m$ and is of the order of a segment length.

KEY WORDS Interfacial Tension / Interface Thickness / Transition / Polymer / Non-Uniform System / Mixture / Solution / Critical Phenomena /
\end{abstract}

Studies on the interface profile and the interfacial tension between demixed liquid phases containing polymers have not been performed extensively. Langhammer and Nestler ${ }^{1}$ measured the interfacial tension of demixed polymer solutions of polymer-polymer-solvent systems, and found very low values of interfacial tension, in the range of $10^{-4}-10^{-2} \mathrm{dyn} / \mathrm{cm}$. Morozova and Krotova, ${ }^{2}$ Gromav and Chalykh, ${ }^{3}$ Voyutskii, Kamenskii, and Fodiman, ${ }^{4}$ Letz, et $a l .{ }^{5}$ measured the thickness of the interfacial layer of polymer-polymer systems with phasecontrast microscopes or electronmicroscopes, and found very large values of the interface thickness, in the range of $10^{2}-10^{5} \mathrm{~nm}$ depending on the combination of polymers. It is not clear, however, whether the diffused interfaces measured were truly equilibrium interfaces or not. On the other hand, $\mathrm{Vrij}^{6}$ derived the equation for the interfacial tension between demixed polymer solutions of symmetrical systems on the basis of the theory of Cahn and Hilliard, ${ }^{7}$ and found qualitative agreement with the experiments of Langhammer and Nestler.
Helfand and Tagami ${ }^{8}$ presented the theory of interfaces of immiscible symmetrical polymer mixtures employing a mean field theory which involved a selfconsistent field calculation, and Helfand and Sapse ${ }^{9}$ extended it to be applicable to asymmetrical systems. They predicted the thickness of a polymer-polymer interface to be of the order of $1 \mathrm{~nm}$ in most cases, depending on the interaction parameter $\chi$. Recently, $\operatorname{Roe}^{10}$ presented the theory for the interface between polymers and polymer solutions based on his own theory for non-uniform systems, which was derived by modifying the Flory-Huggins solution theory. The interfacial tension and interface thickness were evaluated as a function of $\chi-\chi_{c}$ and the chain length, where $\chi_{\mathrm{c}}$ was the value of $\chi$ at the critical temperature.

Generally speaking, the thickness of the interface strongly depends on the temperature $T$, especially near the critical temperature $T_{\mathrm{c}}$. Therefore, the thickness of the interface layer should be discussed as a function of temperature or of $T / T_{\mathrm{c}}$. Previous works except the recent theory of Roe, ${ }^{10}$ however, did not treat 
the temperature dependence sufficiently. In a polymer system, the conformation or dimension of polymer coils at the interface may also be an essentially important problem, which may affect the interface profile and interfacial tension. In Roe's theory, the entropy arising from a distortion of polymer molecules from their equilibrium phase was taken into account by evaluating the possible difference in the distribution of chain ends in the interface from that of interior segments. In Vrij's theory, however, the segment distribution of a polymer chain is assumed to be invariable even at the interface.

In this paper, equations will be derived for the interfacial tension and the quantities concerned with the interface profile, such as the concentration gradient and the dimension of chain molecules at the interface, on the basis of the theory of Cahn and Hilliard, taking into account the change in the dimension of polymer coils at the interface. Interactions between polymers (or a polymer and solvents) are attributed to segment-segment (or segmentsolvent) interactions, and the large interaction range of a polymer molecule is due to the large dimension of a chain coil. In other words, polymer molecules can interpenetrate and have no hard core at the center of a molecule but each segment has a hard core and occupies the volume of a system. In the present theory, therefore, the local number density of segments (or local volume fraction of segments) and the local number density of molecules are distinctively defined and are related to each other through the concentration gradient in a nonuniform system. This idea was first introduced by Vrij and Esker ${ }^{11}$ in their recent theory for critical opalescence of polymer solutions. The present theory is for binary systems including asymmetrical systems. The main aims of the paper are to see what are expected for the changes in the concentration gradient and the dimension of a chain at the interface upon decreasing temperature from a critical temperature, and to evaluate the molecular weight dependence of the interfacial tension and the interface thickness in polymer-solvent binary systems and symmetrical polymer mixtures.

\section{THEORETICAL}

Consider a mixture of $N_{1}$ polymer molecules of species 1 , each consisting of $m_{1}$ segments, and $N_{2}$ polymer molecules of species 2, each consisting of $m_{2}$ segments, in a total volume $V$. Thus, we can write

$$
V=\omega_{1} m_{1} N_{1}+\omega_{2} m_{2} N_{2}
$$

where $\omega_{i}$ is the volume of a segment of species $i$. We assume $\omega_{i}$ to be constant by neglecting the volume change on mixing. The system is demixed into two liquid phases, having a concentration gradient in the $z$-direction. The interface, which is perpendicular to the $z$-direction, has an area $A$. The interfacial tension $\sigma$ is given as the surface excess free energy per unit surface area

$$
\begin{aligned}
\sigma & =\left(F-F^{\prime}\right) / A \\
& =\left\{\left(F-F_{0}\right)-\left(F^{\prime}-F_{0}\right)\right\} / A
\end{aligned}
$$

where $F$ is the Helmholtz free energy of the mixture, $F^{\prime}$ is that of a fictitious system without interface containing two homogeneous phases with the same composition as in the two homogeneous phases of the real system, and $F_{0}$ is the free energy of the pure components before mixing. $F^{\prime}-F_{0}$ is expressed by

$$
F^{\prime}-F_{0}=N_{1}\left\{\mu_{1}(e)-\mu_{1}^{0}\right\}+N_{2}\left\{\mu_{2}(e)-\mu_{2}^{0}\right\}
$$

where $\mu_{i}(e)$ is the chemical potential of species $i$ in phase equilibrium, and $\mu_{i}{ }^{0}$ is that of a pure component $i$.

As pointed out in the introduction, we define the local number density of segments and the local number density of molecules, and relate them to each other. Let the numbers of segments and molecules of species $i$ per unit volume be denoted by $n_{i}$ and $c_{i}$ respectively, which are both given as a function of the coordinate $z$ along which the concentration gradient exists, i.e., $n_{i}(z)$ and $c_{i}(z)$. Since a segment with a hard core occupies the volume of the system, we have

$$
n_{1} \omega_{1}+n_{2} \omega_{2}=1
$$

In general, the relation* of $c_{1} m_{1} \omega_{1}+c_{2} m_{2} \omega_{2}=1$

* In Vrij's theory ${ }^{6}$ for interfacial tension this relation was used, although in the recent theory of Vrij and Esker ${ }^{11}$ for critical opalescence eq 4 was adopted. 
does not always hold, because $n_{i}=m_{i} c_{i}$ is not always satisfied under the existence of a concentration gradient. Designating the segment density of a molecule of species $i$ whose center is located at a position $z$ by $\rho_{i}(r ; z)$ as a function of the distance $r$ from the center of the molecule, $n_{i}$ is expressed in terms of $c_{i}$ by the equation

$$
n_{i}(z)=\int_{-\infty}^{\infty} c_{i}\left(z+r_{z}\right) \rho_{i}\left(-\boldsymbol{r} ; z+r_{z}\right) \mathrm{d} r_{x} \mathrm{~d} r_{y} \mathrm{~d} r_{z}
$$

where $r_{x}, r_{y}$, and $r_{z}$ are the $x, y$, and $z$ components of $\boldsymbol{r}$, respectively, and the center of a molecule is defined by

$$
\begin{array}{r}
\int_{-\infty}^{\infty} \int_{-\infty}^{\infty} \mathrm{d} r_{x} \mathrm{~d} r_{y} \int_{0}^{\infty} \rho \mathrm{d} r_{z}=\int_{-\infty}^{\infty} \int_{-\infty}^{\infty} \mathrm{d} r_{y} \mathrm{~d} r_{z} \int_{0}^{\infty} \rho \mathrm{d} r_{x} \\
=\int_{-\infty}^{\infty} \int_{-\infty}^{\infty} \mathrm{d} r_{z} \mathrm{~d} r_{x} \int_{0}^{\infty} \rho \mathrm{d} r_{y}=\int_{\infty-}^{\infty} \rho \mathrm{d} r_{x} \mathrm{~d} r_{y} \mathrm{~d} r_{z} / 2
\end{array}
$$

The segment density $\rho_{i 0}$ in a uniform system may be reasonably assumed to be Gaussian; i.e., ${ }^{12}$

$$
\rho_{i 0}\left(r_{x}, r_{y}, r_{z}\right)=\left(m_{i} / a^{3} \pi^{3 / 2}\right) \exp \left\{-\left(r_{x}{ }^{2}+r_{y}{ }^{2}+r_{z}^{2}\right) / a_{i}{ }^{2}\right\}
$$

The parameter $a_{i}$ is a measure of the dimension of a polymer coil. In a non-uniform system or in a liquid-liquid interface, however, it is a very difficult problem to determine the segment density rigorously, although many theoretical treatments have been presented for an isolated polymer chain on a rigid surface. Thus, in the present theory we introduce the local expansion coeffecient $\alpha$ of a chain as a function of the position and assume that when a part of a chain is at a position $(x, y, z)$, its dimension is expanded by $\alpha_{x}(x, y, z), \alpha_{y}(x, y, z)$, and $\alpha_{z}(x, y, z)$ times of that of unperturbed state in the directions of $x, y$, and $z$, respectively. Then it follows that

$$
\begin{aligned}
& \rho_{i}(\boldsymbol{r} ; x, y, z) \mathrm{d} r_{x} \mathrm{~d} r_{y} \mathrm{~d} r_{z} \\
& \quad=\rho_{i 0}\left(r_{x} / \bar{\alpha}_{x}, r_{y} / \bar{\alpha}_{y}, r_{z} / \bar{\alpha}_{z}\right) \mathrm{d} r_{x} \mathrm{~d} r_{y} \mathrm{~d} r_{z} /\left(\alpha_{x} \alpha_{y} \alpha_{z}\right)
\end{aligned}
$$

where

$$
r_{x} / \bar{\alpha}_{x}=\int_{0}^{r_{x}} \mathrm{~d} r_{x}{ }^{\prime} / \alpha_{x}, \quad r_{y} / \bar{\alpha}_{y}=\int_{0}^{r_{y}} \mathrm{~d} r_{y}^{\prime} / \alpha_{y}
$$

and

$$
r_{z} / \bar{\alpha}_{z}=\int_{0}^{r_{z}} \mathrm{~d} r_{z}^{\prime} / \alpha_{z}
$$

and $\alpha_{x}, \alpha_{y}$, and $\alpha_{z}$ in eq 8 are expansion factors at $\boldsymbol{r}^{\prime}$. In the present case, since the system has no concentration gradient in the $x$ and $y$-directions, $\alpha_{x}$ and $\alpha_{y}$ are both unity. ${ }^{*}$ Therefore, eq 7 and 8 are written as

$$
\begin{gathered}
\rho_{i}(\boldsymbol{r} ; z) \mathrm{d} r_{x} \mathrm{~d} r_{y} \mathrm{~d} r_{z}=\rho_{i 0}\left(r_{x}, r_{y}, r_{z} / \bar{\alpha}\right) \mathrm{d} r_{x} \mathrm{~d} r_{y} \mathrm{~d} r_{z} / \alpha \\
\bar{\alpha}=r_{z} /\left\{\int_{0}^{r_{z}} \mathrm{~d} r_{z}^{\prime} / \alpha\left(z+r_{z}{ }^{\prime}\right)\right\}
\end{gathered}
$$

where $\alpha_{z}$ is a function of $z$ and is replaced by $\alpha$ for simplicity. Now, assuming that $F$ is separately expressed by the sum of the free energy $\bar{F}$ associated with intermolecular and intersegmental interactions or liquidlike properties, and the free energy $F^{\mathrm{e}}$ associated with chain conformation or elasticity of a polymer coil, one may write

$$
\begin{aligned}
F-F_{0} & =\left(\bar{F}-\bar{F}_{0}\right)+\left(F^{\mathrm{e}}-F_{0}{ }^{\mathrm{e}}\right) \\
& =\left(\bar{U}-\bar{U}_{0}\right)-T\left(\bar{S}-\bar{S}_{0}\right)+\left(F^{\mathrm{e}}-F_{0}{ }^{\mathrm{e}}\right)
\end{aligned}
$$

where $\bar{U}$ is the internal energy arising from the potential energy of the intersegmental interactions and $\bar{S}$ is the entropy in $\bar{F}$ (excluding the entropy associated with chain conformation).

For $\bar{S}-\bar{S}_{0}$, the following expression is assumed $^{* *}$

$$
\bar{S}-\bar{S}_{0}=-k A \int \mathrm{d} z\left(c_{1} \ln \phi_{1}+c_{2} \ln \phi_{2}\right)
$$

where $k$ is the Boltzmann constant, and $\phi_{i}$ denotes the concentration of species $i$ in volume fraction at a position $z$

$$
\phi_{i}=\frac{n_{i} \omega_{i}}{n_{1} \omega_{1}+n_{2} \omega_{2}}=n_{i} \omega_{i}
$$

because of eq 4 . The integration in eq 12 is taken across the whole system. Equation 12

* The following derivation can a be performed generally by putting $\alpha_{x}$ and $\alpha_{y}$ as a function of $(x, y, z)$; thus we can obtain $\alpha_{x}=\alpha_{y}=1$ and find that $\alpha_{z}$ depends only on $z$ but not on $x$ and $y$. However, we put $\alpha_{x}=\alpha_{y}=1$ at the first stage (here) for more simplicity in the derivation.

** Vrij and Esker ${ }^{11}$ used a different expression: $\bar{S}-\overline{S_{0}}=-k A \int \mathrm{d} z\left\{c_{1} \ln \left(c_{1} m_{1} \omega_{1}\right)+c_{2} \ln \left(c_{2} m_{2} \omega_{2}\right)\right\}$ instead of eq 12. The two expressions, however, are easily proved to be equivalent to each other in the present theory, using eq 25 and neglecting the higher (more than second) order derivatives. 
is a well-known approximate formula for the entropy of mixing if applied to a homogeneous mixture. It is assumed that $\bar{S}-\bar{S}_{0}$ can be approximated by the same formula as that of a homogeneous mixture without depending on the concentration gradient explicitly.

For the evaluation of $\bar{U}$, the potential energy of the system is assumed to be the sum of the pairwise potential energy between segments. Then, $\bar{U}$ is expressed by

$$
2 \bar{U}=A \int \mathrm{d} z\left(n_{1} e_{11}+n_{1} e_{12}+n_{2} e_{21}+n_{2} e_{22}\right)
$$

where $e_{i j}$ is the potential energy of a central segment of species $i$ at a position $z$ due to the surrounding segments of species $j$, and is written as

$$
e_{i j}(z)=-\int \varepsilon_{i j}(r) n_{j}\left(z+r_{z}\right) \mathrm{d} r_{x} \mathrm{~d} r_{y} \mathrm{~d} r_{z}
$$

Here, $-\varepsilon_{i j}(r)$ is the potential energy between segments of species $i$ and $j$ separated by $r$.

In order to evaluate $F^{e}$, we must know the conformational free energy $f^{e}$ for a polymer molecule having the segment density represented by eq 7 or 9 , but we don't have a conventional way to evaluate it. When a chain molecule is uniformly expanded, i.e., when $\alpha_{x}, \alpha_{y}$, and $\alpha_{z}$ are independent of the position $r^{\prime}$ in eq 8 or $10, f_{\mathrm{u}}^{\mathrm{e}}-f_{\mathrm{v} 0}^{\mathrm{e}}$ for a uniform expansion may be reasonably given by ${ }^{12}$

$$
f_{\mathrm{u}}^{\mathrm{e}}-f_{\mathrm{u} 0}^{\mathrm{e}}=\frac{k T}{2} \sum_{k=x y z}\left\{\left(\alpha_{k}^{2}-1\right)-\ln \alpha_{k}^{2}\right\}
$$

Then, it is assumed here that $f_{i}^{\mathrm{e}}-f_{i 0}^{\mathrm{e}}$ of species $i$ is approximated by

$f_{i}^{\mathrm{e}}-f_{i 0}^{\mathrm{e}}$

$$
=\frac{k T}{2} \int \frac{\rho_{i}}{m_{i}} \sum_{k=x y z}\left\{\left(\alpha_{k}^{2}-1\right)-\ln \alpha_{k}^{2}\right\} \mathrm{d} r_{x} \mathrm{~d} r_{y} \mathrm{~d} r_{z}
$$

If $\alpha_{k}$ is independent of $\boldsymbol{r}$, i.e., in the case of uniform expansion, eq 17 becomes eq 16 . Therefore, eq 17 may be a good approximation for a small concentration gradient. In the present case of $\alpha_{x}=\alpha_{y}=1$, eq 17 is written as

$$
\begin{aligned}
f_{i}{ }^{\mathrm{e}}-f_{i 0}^{\mathrm{e}}= & \frac{k T}{2} \int \frac{\rho_{i}(\boldsymbol{r} ; z)}{m_{i}}\left[\left\{\alpha_{i}\left(z+r_{z}\right)\right\}^{2}\right. \\
& \left.-1-\ln \left\{\alpha_{i}\left(z+r_{z}\right)\right\}^{2}\right] \mathrm{d} r_{x} \mathrm{~d} r_{y} \mathrm{~d} r_{z}
\end{aligned}
$$

Accordingly, it follows with the aid of eq 18 and 5 that

$$
\begin{aligned}
F^{\mathrm{e}}-F_{0}{ }^{\mathrm{e}}= & A \int \mathrm{d} z \sum_{i=12}\left(f_{i}^{\mathrm{e}}-f_{i 0}^{\mathrm{e}}\right) c_{i}(z) \\
= & A \int \mathrm{d} z \frac{k T}{2} \sum_{i=12} \int\left\{\alpha_{i}(z)^{2}-1-\ln \alpha_{i}(z)^{2}\right\} \\
& \times \rho_{i}\left(r ; z-r_{z}\right) c_{i}\left(z-r_{z}\right) / m_{i} \mathrm{~d} r_{x} \mathrm{~d} r_{y} \mathrm{~d} r_{z} \\
= & A \int \mathrm{d} z \frac{k T}{2} \sum_{i=12}\left(\alpha_{i}{ }^{2}-1-\ln \alpha_{i}{ }^{2}\right) \\
& \times \int \rho_{i}\left(-\boldsymbol{r} ; z+r_{z}\right) c_{i}\left(z+r_{z}\right) / m_{i} \mathrm{~d} r_{x} \mathrm{~d} r_{y} \mathrm{~d} r_{z} \\
= & A \int \mathrm{d} z \frac{k T}{2} \sum_{i=12} \frac{n_{i}}{m_{i}}\left\{\left(\alpha_{i}{ }^{2}-1\right)-\ln \alpha_{i}{ }^{2}\right\}
\end{aligned}
$$

The free energy $F-F_{0}$ of the non-uniform system considered is now expressed by eq 11 with eq 12,14, and 19. Therefore, if eq 5 and 15 with eq 9 and 10 are expanded in a Taylor series about a position $z, F-F_{0}$ will be expressed by a local free energy depending on the local composition $(n)$, expansion factor $(\alpha)$, and the gradients of $n$ and $\alpha$, as in the Cahn-Hilliard theory. The derivatives of higher order (third and more) are neglected in the present theory. Equation 15 is written in an expansion form as

$$
\begin{aligned}
e_{i j} & =-\int \varepsilon_{i j}(r)\left\{n_{j}(z)+r_{z} \dot{n}_{j}+r_{z}^{2} \ddot{n}_{j} / 2+\cdots\right\} \mathrm{d} r_{x} \mathrm{~d} r_{y} \mathrm{~d} r_{z} \\
& =-n_{j}(z) W_{i j}-\ddot{n}_{j} l_{i j}^{2} W_{i j} / 6+\cdots
\end{aligned}
$$

where

$$
\begin{aligned}
W_{i j} & =\int \varepsilon_{i j}(r) \mathrm{d} r_{x} \mathrm{~d} r_{y} \mathrm{~d} r_{z} \\
l_{i j}{ }^{2} & =3 \int r_{z}{ }^{2} \varepsilon_{i j}(r) \mathrm{d} r_{x} \mathrm{~d} r_{y} \mathrm{~d} r_{z} / W_{i j} \\
& =\int r^{2} \varepsilon_{i j}(r) \mathrm{d} r_{x} \mathrm{~d} r_{y} \mathrm{~d} r_{z} / \int \varepsilon_{i j}(r) \mathrm{d} r_{x} \mathrm{~d} r_{y} \mathrm{~d} r_{z}
\end{aligned}
$$

and the dots indicate the differentiation with respect to $z$.

Substitution of eq 9 with eq 6 into eq 5 gives

$$
\begin{aligned}
n_{i}= & \frac{m_{i}}{a_{i} \alpha_{i} \pi^{1 / 2}} \int_{-\infty}^{\infty} \exp \left\{-\frac{r_{z}{ }^{2}}{a_{i}{ }^{2} \bar{\alpha}_{i}{ }^{2}}\right\} \\
& \times\left[c_{i}+\dot{c}_{i} r_{z}+\frac{\ddot{c}_{i} r_{z}{ }^{2}}{2}+\cdots\right] \mathrm{d} r_{z}
\end{aligned}
$$

The term of $\exp \left(-r_{z}{ }^{2} / a_{i}{ }^{2} \bar{\alpha}_{i}{ }^{2}\right)$ in eq 22 is effective in the integration only when $r_{z}{ }^{2} / a_{i}{ }^{2} \bar{\alpha}_{i}{ }^{2}$ is almost equal to or less than unity. Using this 
fact and eq 10, and neglecting the higher order terms, the exponential term in eq 22 is approximated by

$$
\begin{aligned}
& \exp \left(-\frac{r_{z}{ }^{2}}{a_{i}{ }^{2} \bar{\alpha}_{i}{ }^{2}}\right) \simeq\left\{\exp \left(-\frac{r_{z}{ }^{2}}{a_{i}{ }^{2} \alpha_{i}{ }^{2}(z)}\right)\right\} \\
& \quad \times\left\{1+A(\alpha, \dot{\alpha}) r_{z}+B(\alpha, \dot{\alpha}, \ddot{\alpha}) r_{z}{ }^{4}+c\left(\dot{\alpha}^{2}\right) r_{z}{ }^{6}\right\}
\end{aligned}
$$

(see Appendix I). Substituting eq 23 into eq 22 and performing the integration in eq 22 , we have

$$
\begin{aligned}
n_{i}= & m_{i} c_{i}+m_{i} a_{i}{ }^{2} \alpha_{i}{ }^{2} \ddot{c}_{i} / 4+3 m_{i} a_{i}{ }^{2} \alpha_{i} \dot{c}_{i} \dot{\alpha}_{i} / 4 \\
& +m_{i} a_{i}{ }^{2} c_{i} \dot{\alpha}_{i}{ }^{2} / 4+m_{i} a_{i}{ }^{2} \alpha_{i} c_{i} \ddot{\alpha}_{i} / 4
\end{aligned}
$$

or

$$
\begin{aligned}
n_{i}= & m_{i} c_{i}+a_{i}{ }^{2} \alpha_{i}{ }^{2} \ddot{n}_{i} / 4+3 a_{i}{ }^{2} \alpha_{i} \dot{n}_{i} \dot{\alpha}_{i} / 4 \\
& +a_{i}{ }^{2} n_{i} \dot{\alpha}_{i}{ }^{2} / 4+a_{i}{ }^{2} \alpha_{i} n_{i} \ddot{\alpha}_{i} / 4
\end{aligned}
$$

neglecting the higher order terms.

An expression for $\bar{U}$ is given by substitution of eq 20 and 25 into 14 , and $\bar{U}_{0}$ is derived by putting $\dot{n}_{i}, \ddot{n}_{i}, \dot{\alpha}_{i}$, and $\ddot{\alpha}_{i}=0$ in the expression of $\bar{U}$ as $2 \bar{U}_{0}=-2 A \int\left(n_{1} W_{11} / \omega_{1}+n_{2} W_{22} / \omega_{2}\right) \mathrm{d} z$. From these expressions of $\bar{U}$ and $\bar{U}_{0}$, and eq 11 , 12 , and 19 , one has the following equation for $F-F_{0}$;

$$
F-F_{0}=A \int\left(f+h{\dot{\phi_{1}}}^{2}+\frac{q_{1}}{2} \dot{\phi}_{1} \dot{\gamma}_{1}+\frac{q_{2}}{2} \dot{\phi}_{2} \dot{\gamma}_{2}\right) \mathrm{d} z
$$

where

$$
\begin{gathered}
f=f_{\mathrm{u}}+\frac{k T}{2} \sum_{i=1,2} \frac{\phi_{i}}{m_{i} \omega_{i}}\left\{\left(\gamma_{i}-1\right)-\ln \gamma_{i}\right\} \\
f_{\mathrm{u}}=E\left(\phi_{1}-\phi_{1}{ }^{2}\right)+k T \sum_{i=1,2} \frac{\phi_{i}}{m_{i} \omega_{i}} \ln \phi_{i} \\
E=\frac{1}{2}\left(\frac{W_{11}}{\omega_{1}{ }^{2}}-\frac{2 W_{12}}{\omega_{1} \omega_{2}}+\frac{W_{22}}{\omega_{2}{ }^{2}}\right) \\
\gamma_{i}=\alpha_{i}{ }^{2} \\
h=\bar{\Omega}+\frac{q_{1} \gamma_{1}}{\phi_{1}}+\frac{q_{2} \gamma_{2}}{\phi_{2}} \\
q_{i}=\frac{a_{i}{ }^{2} k T}{4 m_{i} \omega_{i}}
\end{gathered}
$$

and

$$
\bar{\Omega}=\frac{1}{12}\left(\frac{W_{11}}{\omega_{1}^{2}} l_{11}^{2}-\frac{2 W_{12}}{\omega_{1} \omega_{2}} l_{12}^{2}+\frac{W_{22}}{\omega_{2}^{2}} l_{22}^{2}\right)
$$

In obtaining eq 26 , we used the relation $\int g \ddot{\phi} \mathrm{d} z=-\int \dot{g} \dot{\phi} \mathrm{d} z$ and $\int g \ddot{\gamma} \mathrm{d} z=-\int \dot{g} \dot{\gamma} \mathrm{d} z$ (where $g$ is a function of $z$ ) because we can chose the system so that $\dot{\phi}=\dot{\gamma}=0$ at its boundary. As seen from the form of eq 28 , eq 26 is the wellknown formula for the free energy of mixing in the Flory-Huggins solution theory if applied to a homogeneous system in which $\dot{\gamma}=\dot{\phi}=0$ and $\gamma=1$.

The chemical potential $\mu_{i}(e)-\mu_{i}^{0}$ in eq 3 can be evaluated by

$$
\begin{aligned}
\mu_{i}(e) & -\mu_{i}{ }^{0}=\partial\left[f_{\mathrm{u}}(e) V\right] / \partial N_{i} \\
& =\omega_{i} m_{i} f_{\mathrm{u}}(e)+m_{i} \omega_{i}\left\{1-\phi_{i}(e)\right\}\left(\partial f_{\mathrm{u}} / \partial \phi_{i}\right)_{\mathrm{e}}
\end{aligned}
$$

where eq 1 and 13 were used, and $f_{\mathrm{u}}(e)$ and $\left(\partial f_{\mathrm{u}} / \partial \phi_{i}\right)_{\mathrm{e}}$ are the values of $f_{\mathrm{u}}$ and $\left(\partial f_{\mathrm{u}} / \partial \phi_{i}\right)$ at the compositions $\phi_{i}(e)$ of homogeneous phases in equilibrium. Then, it follows from eq 3 and 34 that

$$
\begin{aligned}
F-F_{0}= & A \int \mathrm{d} z\left[n_{1}\left\{\mu_{1}(e)-\mu_{1}^{0}\right\} / m_{1}\right. \\
& \left.+n_{2}\left\{\mu_{2}(e)-\mu_{2}^{0}\right\} / m_{2}\right] \\
= & A \int \mathrm{d} z\left[f_{\mathrm{u}}(e)+\left\{\phi_{1}-\phi_{1}(e)\right\}\left(\partial f_{\mathrm{u}} / \partial \phi_{1}\right)_{\mathrm{e}}\right]
\end{aligned}
$$

Finally, from eq 2, 26, and 35 with eq 27 and 28 , we obtain

$$
\sigma=\int \mathrm{d} z\left(\Delta f+h \dot{\phi}_{1}{ }^{2}+\frac{q_{1}}{2} \dot{\phi}_{1} \dot{\gamma}_{1}+\frac{q_{2}}{2} \dot{\phi}_{2} \dot{\gamma}_{2}\right)
$$

where

$$
\begin{gathered}
\Delta f=\Delta f_{\mathrm{u}}+\Delta f^{\mathrm{e}} \\
\Delta f_{\mathrm{u}}=E\left\{\phi_{1}-\phi_{1}(e)\right\}^{2} \\
+k T\left\{\frac{\phi_{1}}{m_{1} \omega_{1}} \ln \left(\phi_{1} / \phi_{1}(e)\right)+\frac{\phi_{2}}{m_{2} \omega_{2}} \ln \left(\phi_{2} / \phi_{2}(e)\right)\right. \\
\left.+\left(\phi_{1}-\phi_{1}(e)\right)\left(\frac{1}{m_{2} \omega_{2}}-\frac{1}{m_{1} \omega_{1}}\right)\right\} \\
\Delta f^{e}=\frac{k T}{2}\left\{\frac{\phi_{1}}{m_{1} \omega_{1}}\left(\gamma_{1}-1-\ln \gamma_{1}\right)\right. \\
\left.+\frac{\phi_{2}}{m_{2} \omega_{2}}\left(\gamma_{2}-1-\ln \gamma_{2}\right)\right\}
\end{gathered}
$$

The equilibrium concentration $\dot{\phi}_{i}(e)$ can be determined by the condition that the chemical potentials $\mu_{i}(e)$ of the two demixed phases are equal to each other, using eq 28 and 34 . That is, $\phi_{1}(e)\left(=1-\phi_{2}(e)\right)$ is given as a solution of the simultaneous equations 
Theory of Liquid-Liquid Interface of Polymer Systems

$$
\begin{array}{rl}
m_{1} \omega_{1} & E\left\{1-\phi_{1}{ }^{\prime}(e)\right\}^{2} \\
& +k T\left[\ln \phi_{1}^{\prime}(e)+\left\{1-\phi_{1}{ }^{\prime}(e)\right\}\left(1-\frac{m_{1} \omega_{1}}{m_{2} \omega_{2}}\right)\right] \\
= & m_{1} \omega_{1} E\left\{1-\phi_{1}{ }^{\prime \prime}(e)\right\}^{2} \\
+ & k T\left[\ln \phi_{1}^{\prime \prime}(e)+\left\{1-\phi_{1}{ }^{\prime \prime}(e)\right\}\left(1-\frac{m_{1} \omega_{1}}{m_{2} \omega_{2}}\right)\right]
\end{array}
$$

and

$$
\begin{aligned}
m_{2} \omega_{2} E \phi_{1}{ }^{\prime 2}(e) & \\
+ & k T\left[\ln \left\{1-{\phi_{1}}^{\prime}(e)\right\}+{\phi_{1}}^{\prime}(e)\left(1-\frac{m_{2} \omega_{2}}{m_{1} \omega_{1}}\right)\right] \\
= & m_{2} \omega_{2} E \phi_{1}{ }^{\prime \prime 2}(e) \\
+ & k T\left[\ln \left\{1-{\phi_{1}}^{\prime \prime}(e)\right\}+{\phi_{1}}^{\prime \prime}(e)\left(1-\frac{m_{2} \omega_{2}}{m_{1} \omega_{1}}\right)\right]
\end{aligned}
$$

where $\phi_{1}{ }^{\prime}(e)$ and $\phi_{1}{ }^{\prime \prime}(e)$ are the equilibrium concentrations $\phi_{1}(e)$ of the two demixed phases.

At equilibrium, the variations of the composition $\phi$ and the expansion factor $\alpha$ will be such that the integral in eq 36 is a minimum. Then, using the variational method, the Euler equations are

$$
\begin{aligned}
& \left(\partial I / \partial \phi_{1}\right)-\frac{\mathrm{d}}{\mathrm{d} z}\left(\partial I / \partial \dot{\phi}_{1}\right)=0 \\
& \left(\partial I / \partial \gamma_{i}\right)-\frac{\mathrm{d}}{\mathrm{d} z}\left(\partial I / \partial \dot{\gamma}_{i}\right)=0
\end{aligned}
$$

or

$$
\frac{\mathrm{d}}{\mathrm{d} z}\left(I-\dot{\phi}_{1} \frac{\partial I}{\partial \dot{\phi}_{1}}-\dot{\gamma}_{1} \frac{\partial I}{\partial \dot{\gamma}_{1}}-\dot{\gamma}_{2} \frac{\partial I}{\partial \dot{\gamma}_{2}}\right)=0
$$

because the integrand $I$ in eq 36 is a function of $\phi_{1}, \gamma_{1}, \gamma_{2}, \dot{\phi}_{1}, \dot{\gamma}_{1}$, and $\dot{\gamma}_{2}$, and does not explicitly depend on $z$, i.e., $\partial I / \partial z=0$. Since all of $\Delta f, \phi_{1}, \dot{\gamma}_{1}$, and $\dot{\gamma}_{2}$ tend to zero as $z \rightarrow \pm \infty$ (at the boundary of the system), eq 44 leads to

$$
I=\phi_{1} \frac{\partial I}{\partial \dot{\phi}_{1}}+\dot{\gamma}_{1} \frac{\partial I}{\partial \dot{\gamma}_{1}}+\dot{\gamma}_{2} \frac{\partial I}{\partial \dot{\gamma}_{2}}
$$

Substituting the explicit form of the integrand $I$ in eq 36 into eq 42, 43, and 45 gives

$$
\begin{gathered}
\partial \Delta f / \partial \phi_{1}=2\left(\dot{h} \dot{\phi}_{1}+h \ddot{\phi}_{1}\right)+q_{1} \ddot{\gamma}_{1} / 2-q_{2} \ddot{\gamma}_{2} / 2 \\
\partial \Delta f^{\mathrm{e}} / \partial \gamma_{i}=-q_{i} \dot{\phi}_{i}{ }^{2} / \phi_{i}+q_{i} \ddot{\phi}_{i} / 2
\end{gathered}
$$

and

$$
\begin{aligned}
\Delta f & =h \dot{\phi}_{1}{ }^{2}+\frac{q_{1}}{2} \dot{\phi}_{1} \dot{\gamma}_{1}-\frac{q_{2}}{2} \dot{\phi}_{1} \dot{\gamma}_{2} \\
& =H \dot{\phi}_{1}{ }^{2}
\end{aligned}
$$

where $H$ is defined by

$$
H=h+\frac{q_{1} \dot{\gamma}_{1}}{2 \dot{\phi}_{1}}-\frac{q_{2} \dot{\gamma}_{2}}{2 \dot{\phi}_{1}}
$$

From eq 36 and 48, we have the following expression for the interfacial tension $\sigma$

$$
\begin{aligned}
\sigma & =2 \int \Delta f \mathrm{~d} z \\
& =2 \int_{\phi_{1^{\prime}}(e)}^{\phi_{1^{\prime \prime}}(e)}(H \Delta f)^{1 / 2} \mathrm{~d} \phi_{1}
\end{aligned}
$$

In principle, the simultaneous differential eq 46,47 , or 48 afford the composition and the chain dimension profile, i.e., the variations of $\phi_{1}, \gamma_{1}$, and $\gamma_{2}$ with $z$, and substitution of these results into eq 50 gives the interfacial tension $\sigma$.

Behavior Near Critical Temperature $T_{\mathrm{c}}$

Since $\Delta f_{\mathrm{u}}$ given by eq 38 can be expanded in a Taylor series about $T_{\mathrm{c}}$ and critical concentration $\phi_{1 \mathrm{c}}$, we have the following expression for $\Delta f_{\mathrm{u}}$ near $T_{\mathrm{c}} ;^{7}$

$$
\Delta f_{\mathrm{u}}=\kappa\left\{\left(\Delta \phi_{\mathrm{e}}\right)^{2}-(\Delta \phi)^{2}\right\}^{2}
$$

where

$$
\begin{gathered}
\left(\Delta \phi_{\mathrm{e}}\right)^{2}=\beta\left(T_{\mathrm{c}}-T\right) / 2 \kappa \\
\beta \equiv\left(\partial^{3} f_{\mathrm{u}} / \partial T \partial \phi_{1}{ }^{2}\right) / 2 !
\end{gathered}
$$

and

$$
\kappa \equiv\left(\partial^{4} f_{\mathrm{u}} / \partial \phi_{1}{ }^{4}\right) / 4 !
$$

Here, $\quad \Delta \phi_{\mathrm{e}}=\phi_{1 \mathrm{c}}-\phi_{1}{ }^{\prime}(e)=\phi_{1}{ }^{\prime \prime}(e)-\phi_{1 \mathrm{c}}\left[\phi_{1}{ }^{\prime \prime}(e)>\right.$ $\left.\phi_{1}^{\prime}(e)\right]$, and $\Delta \phi=\phi_{1}-\phi_{1 c}$. Since $\gamma_{i}$ may be very close to unity near $T_{\mathrm{c}}, \Delta f$ may be expressed from eq $37-39$ as

$$
\Delta f=\Delta f_{\mathrm{u}}+O\left[\frac{k T_{\mathrm{c}} \phi_{i \mathrm{c}}}{m_{i} \omega_{i}}\left(1-\gamma_{i}\right)^{2}\right]
$$

On the other hand, eq 47 gives

$$
1-\gamma_{i}=O\left[\frac{a_{i}^{2} \dot{\phi}_{1}^{2}}{\phi_{i \mathrm{c}}^{2}}\right]
$$

and

$$
\dot{\gamma}_{i}=O\left[\frac{a_{i}{ }^{2}}{\phi_{i \mathrm{c}}^{3}} \dot{\phi}_{i}{ }^{3}\right]
$$

$H$ defined by eq 49 is then expressed near $T_{\mathrm{c}}$ as

$$
H=h_{\mathrm{c}}+O\left[\frac{a_{i}{ }^{2} k T_{\mathrm{c}}}{\phi_{i \mathrm{c}}^{2} m_{i} \omega_{i}} \Delta \phi\right]+O\left[\frac{a_{i}{ }^{2} k T_{\mathrm{c}}}{4 \phi_{i \mathrm{c}} m_{i} \omega_{i}}\left(1-\gamma_{i}\right)\right]
$$


where

$$
h_{\mathrm{c}}=\bar{\Omega}+\frac{a_{1}^{2} k T_{\mathrm{c}}}{4 \phi_{1 \mathrm{c}} m_{1} \omega_{1}}+\frac{a_{2}{ }^{2} k T_{\mathrm{c}}}{4\left(1-\phi_{1 \mathrm{c}}\right) m_{2} \omega_{2}} .
$$

From eq 55, 56, 58, and 48, we derive

$$
\begin{aligned}
\Delta f & =\Delta f_{\mathrm{u}}+O\left[\frac{k T_{\mathrm{c}}}{m_{i} \omega_{i}} \frac{a_{i}{ }^{4}}{\phi_{i \mathrm{c}}^{3}} \dot{\phi}_{1}{ }^{4}\right] \\
& =\Delta f_{\mathrm{u}}+O\left[\frac{(\Delta f)^{2}}{h_{\mathrm{c}}^{2}} \frac{k T_{\mathrm{z}}}{m_{i} \omega_{i}} \frac{a_{i}{ }^{4}}{\phi_{i \mathrm{c}}^{3}}\right] \\
& =\Delta f_{\mathrm{u}}+O\left[\frac{m_{i} \omega_{i}}{k T_{\mathrm{c}} \phi_{i \mathrm{c}}}\left(\Delta f_{\mathrm{u}}\right)^{2}\right]
\end{aligned}
$$

Therefore, considering $\Delta f_{\mathrm{u}} \rightarrow 0$ when $T \rightarrow T_{\mathrm{c}}$, and neglecting higher order terms, one has $\Delta f=\Delta f_{\mathrm{u}}$ and $H=h_{\mathrm{c}}$ near $T_{\mathrm{c}}$. Then, finally it follows from eq 50 and 51 that

$$
\begin{aligned}
\sigma_{\left(T \sim T_{\mathrm{c}}\right)} & =2 \int_{\phi_{1^{\prime}}(e)}^{\phi_{1}{ }^{\prime \prime}(e)}\left(h_{\mathrm{c}} \Delta f_{\mathrm{u}}\right)^{1 / 2} \mathrm{~d} \phi_{1} \\
& =\frac{2 \sqrt{2}}{3 \kappa} h_{\mathrm{c}}{ }^{1 / 2} \beta^{3 / 2}\left(T_{\mathrm{c}}-T\right)^{3 / 2}
\end{aligned}
$$

substituting eq 28 into eq 53 and 54 to be

$$
\beta=\frac{k}{2 \omega}\left(m_{1}^{-1 / 2}+m_{2}^{-1 / 2}\right)^{2}
$$

and

$$
\kappa=\frac{E}{6}\left(m_{1}^{-1 / 2}+m_{2}^{-1 / 2}\right)^{2}\left(m_{1} m_{2}\right)^{1 / 2}
$$

$T_{\mathrm{c}}$ and $\phi_{1 \mathrm{c}}$ are given from $\partial \mu_{i} / \partial \phi_{i}=\partial^{2} \mu_{i} / \partial \phi_{i}{ }^{2}=0$ with eq 28 and 34, and are evaluated, using eq 38 with $\omega_{1}=\omega_{2}=\omega$, to be

$$
T_{\mathrm{c}}=\frac{2 \omega E}{k}\left(m_{1}^{-1 / 2}+m_{2}^{-1 / 2}\right)^{-2}
$$

and

$$
\phi_{1 \mathrm{c}}=\left\{1+\left(m_{1} / m_{2}\right)^{1 / 2}\right\}^{-1}
$$

Defining

$$
\lambda^{2} \equiv 2 \bar{\Omega} / E
$$

and

$$
b_{i}{ }^{2} \equiv\left(a_{i}^{2} / m_{i}\right) / \lambda^{2}
$$

When $\omega_{1}=\omega_{2}=\omega, \beta$ and $\kappa$ are evaluated by and substituting eq $62-65$ in eq 61 gives

$$
\frac{\sigma}{E \lambda}=\frac{4\left\{1+\left(m_{1} m_{2}\right)^{1 / 2}\left(b_{1}{ }^{2} m_{1}{ }^{1 / 2}+b_{2}{ }^{2} m_{2}{ }^{1 / 2}\right)\left(m_{1}{ }^{1 / 2}+m_{2}{ }^{1 / 2}\right)^{-1}\right\}^{1 / 2}}{\left(m_{1}{ }^{-1 / 2}+m_{2}{ }^{-1 / 2}\right)^{2}\left(m_{1} m_{2}\right)^{1 / 2}}(1-\tilde{T})^{3 / 2}
$$

or

$$
\frac{\sigma \omega}{k T_{\mathrm{c}} \lambda}=\frac{2\left\{1+\left(m_{1} m_{2}\right)^{1 / 2}\left(b_{1}{ }^{2} m_{1}{ }^{1 / 2}+b_{2}{ }^{2} m_{2}{ }^{1 / 2}\right)\left(m_{1}{ }^{1 / 2}+m_{2}{ }^{1 / 2}\right)^{-1}\right\}^{1 / 2}}{\left(m_{1} m_{2}\right)^{1 / 2}}(1-\tilde{T})^{3 / 2}
$$

where a reduced temperature $\tilde{T}$ is defined by $\tilde{T}=T / T_{\mathrm{c}}$. Since $\lambda$ is the interaction length of the segments ${ }^{7}$ and $\left(a_{i}^{2} / m_{i}\right)^{1 / 2}$ is of the order of the length of a segment, $b_{i}$ is of the order of unity. When a symmetrical polymer system is considered, i.e., when $m_{1}=m_{2}=m$ and $b_{1}=b_{2}=b$, eq $68^{\prime}$ becomes

$$
\begin{aligned}
\sigma \omega /\left(k T_{\mathrm{c}} \lambda\right) & =2\left(1+m b^{2}\right)^{1 / 2} m^{-1}(1-\tilde{T})^{3 / 2} \\
& \simeq 2 b m^{-1 / 2}(1-\tilde{T})^{3 / 2}
\end{aligned}
$$

with

$$
T_{\mathrm{c}}=\omega E m / 2 k
$$

and in the case of a polymer solution, i.e., when $m_{1}=m, m_{2}=1, b_{1}=b$, and $b_{2}=0$, eq $68^{\prime}$ becomes

$$
\begin{aligned}
& \sigma \omega /\left(k T_{\mathrm{c}} \lambda\right) \\
& \quad=2\left(b^{2}+m^{-1 / 2}+m^{-1}\right)^{1 / 2}\left(1+m^{1 / 2}\right)^{-1 / 2}(1-\tilde{T})^{3 / 2} \\
& \quad \simeq 2 b m^{-1 / 4}(1-\tilde{T})^{3 / 2}
\end{aligned}
$$

with

$$
T_{\mathrm{c}}=\frac{2 \omega E}{k\left(1+m^{-1 / 2}\right)^{2}}
$$

For a regular solution, $b_{1}=b_{2}=0$ and $m_{1}=m_{2}=1$. Then eq $68^{\prime}$ is

$$
\sigma \omega /\left(k T_{\mathrm{c}} \lambda\right)=2(1-\tilde{T})^{3 / 2}
$$

with

$$
T_{\mathrm{c}}=\omega E / 2 k
$$

Letting the thickness $L$ of interface be defined by

$$
L \equiv\left\{\phi_{1}{ }^{\prime \prime}(e)-\phi_{1}{ }^{\prime}(e)\right\} / \dot{\phi}_{1(\ddot{\phi}=0)}
$$

$L$ near $T_{\mathrm{c}}$ is evaluated as

$$
\begin{aligned}
L= & 2 \Delta \phi_{\mathrm{e}} /\left(\Delta f_{\mathrm{u}} / h_{\mathrm{c}}\right)^{1 / 2} \\
= & 2\left(h_{\mathrm{c}} / T_{\mathrm{c}} \beta\right)^{1 / 2}(1-\tilde{T})^{-1 / 2} \\
= & 2 \lambda\left\{1+\left(m_{1} m_{2}\right)^{1 / 2}\left(b_{1}{ }^{2} m_{1}{ }^{1 / 2}+{b_{2}}^{2}{m_{2}}^{1 / 2}\right)\right. \\
& \left.\times\left(m_{1}{ }^{1 / 2}+m_{2}{ }^{1 / 2}\right)^{-1}\right\}^{1 / 2}(1-\tilde{T})^{-1 / 2}
\end{aligned}
$$


by using eq $48,51-54$, and 59 with eq 66 and 67, and noticing $\Delta f \rightarrow \Delta f_{\mathrm{u}}$ and $H \rightarrow h_{\mathrm{c}}$ near $T_{\mathrm{c}}$. Corresponding to eq $69-71, L$ for the three cases treated above are:

A symmetrical polymer system

$$
\begin{aligned}
L / \lambda & =2\left(1+b^{2} m\right)^{1 / 2}(1-\tilde{T})^{-1 / 2} \\
& \simeq 2 b m^{1 / 2}(1-\tilde{T})^{-1 / 2}
\end{aligned}
$$

A polymer solution (a polymer-solvent system)

$$
\begin{aligned}
L / \lambda & =2\left\{1+m b^{2}\left(1+m^{1 / 2}\right)^{-1}\right\}^{1 / 2}(1-\tilde{T})^{-1 / 2} \\
& \simeq 2 b m^{1 / 4}(1-\tilde{T})^{-1 / 2}
\end{aligned}
$$

A regular solution

$$
L / \lambda=2(1-\tilde{T})^{1 / 2}
$$

Profile of the Interface at $\ddot{\phi}=0$ in Simple Systems Although the simultaneous differential equations of eq 46,47 , and 48 cannot be solved analytically, we can derive from these equations the concentration gradient $\dot{\phi}_{0}$ and the expansion factor $\alpha_{0}$ (and the concentration $\phi_{0}$ ) at $\ddot{\phi}=0$, i.e., at the inflection point of concentration profile, to see the behaviors of the profile of the interface and the interfacial tension as a function of temperature and molecular weight.

From eq 47 with 39 and 32, we obtain

$$
2{\phi_{i}}^{2}\left(1-\gamma_{i}{ }^{-1}\right)-a_{i}{ }^{2} \dot{\phi}_{i}{ }^{2}-\phi_{i} a_{i}{ }^{2} \ddot{\phi}_{i} / 2=0
$$

At $\ddot{\phi}=0$, eq. 77 becomes

$$
\dot{\phi}_{i 0}^{2} / \phi_{i 0}=2\left(\gamma_{i 0}^{-1}-1\right) / a_{i}{ }^{2}
$$

where the subscript 0 indicates the value at the inflection point of the concentration profile. Neglecting the higher order derivatives of $\phi$ than $\dddot{\phi}$, it follows from eq 77 with eq 78 that, at $\ddot{\phi}=0$,

$$
\dot{\gamma}_{i 0}=-2\left(\gamma_{i 0}^{2}-\gamma_{i 0}\right) \dot{\phi}_{i 0} / \phi_{i 0}+a_{i}{ }^{2} \gamma_{i 0}^{2} \dddot{\phi}_{i 0} / 4 \phi_{i 0}
$$

and

$$
\begin{aligned}
\ddot{\gamma}_{0}= & -4\left(\gamma_{i 0}-1\right)^{2}\left(4 \gamma_{i 0}-1\right) / a_{i}{ }^{2} \\
& -a_{i}{ }^{2}{ }^{2}{ }_{i 0}\left(4 \gamma_{i 0}-1\right) \dddot{\phi}_{i 0} \dot{\phi}_{i 0} / 2 \phi_{i 0}{ }^{2}+a_{i}{ }^{4}{ }^{3}{ }^{3}{ }_{i 0} \dddot{\phi}_{i 0}^{2} / 8 \phi_{i 0}^{2}
\end{aligned}
$$

If $\dddot{\phi}_{0}$ can be neglected or can be estimated, $\gamma_{i 0}$, $\phi_{i 0}, \dot{\phi}_{i 0}$, etc., at $\ddot{\phi}=0$ can be obtained from eq 46 and 48 with eq $78-80$. For simplicity, we will deal with following two simple cases:

(1) A symmetrical system of two polymers

Consider a system in which $a_{1}=a_{2}=a, m_{1}=$ $m_{2}=m$, and $\omega_{1}=\omega_{2}=\omega$. From eq 46 ,

$$
\phi_{0}=\frac{1}{2}
$$

is obtained without any approximation. Neglecting the terms of $\dddot{\phi}_{0}$ and substituting eq 78,79 , and 81 in eq 48 gives

$$
\begin{gathered}
\tilde{T}\left\{\left(3-\gamma_{0}\right)\left(1-\gamma_{0}\right)+\ln \gamma_{0}\right\}+(m b)^{-1}\left(\gamma_{0}^{-1}-1\right) \\
+(\tilde{T} / 2) \ln \{2 \phi(e)\}+\phi(e)^{2}-2 \phi(e)+\frac{3}{4}=0
\end{gathered}
$$

where

$$
\begin{gathered}
b^{2}=\left(a^{2} / m\right) / \lambda^{2} \\
\tilde{T}=T / T_{\mathrm{c}}=2 k T /(m \omega E)
\end{gathered}
$$

and the equilibrium concentration $\phi(e)$ can be determined by the equation

$$
\tilde{T} \ln [\phi(e) /\{1-\phi(e)\}]-2\{2 \phi(e)-1\}=0
$$

which is derived from eq 40 or 41 . The value of $\gamma_{0}$, accordingly $\alpha_{0}$, can be obtained from eq 82 , and the value of $\dot{\phi}_{0}$ can be calculated by eq 78 with the $\gamma_{0}$ thus obtained and $\phi_{0}=\frac{1}{2}$ (eq 81). (2) A polymer solution (a polymer-solvent system)

Consider a system in which $m_{1}=m, m_{2}=1$, $a_{1}=a, a_{2}=0$, and $\omega_{1}=\omega_{2}=\omega$. In this case eq 81 does not always hold because the system is an asymmetrical one. Therefore we should solve the simultaneous equations of eq 46 and 48 with respect to $\phi$ and $\gamma$. Although we used the approximation of $\dddot{\phi}_{0}=0$ in the calculation for the symmetrical system, it is not a good approximation in this case because the equations are simultaneous ones and the terms of $\dddot{\phi}_{0}$ are more effective in eq 46 than in eq 48 . Then, we will estimate $\dddot{\phi}_{0}$ in terms of $\dot{\phi}_{0}$. Assuming a hyperbolic tangent function for the concentration profile near the position of $\ddot{\phi}=0, \dddot{\phi}_{0}$ may be approximated by (see Appendix II)

$$
\dddot{\phi_{0}}=-B \dot{\phi}_{0}{ }^{3} / \phi_{0}{ }^{2}
$$

with

$$
B=8 \phi_{0}{ }^{2} /\left\{\phi^{\prime \prime}(e)-\phi^{\prime}(e)\right\}^{2}
$$

Using eq 86 we have the following simultaneous equations, from eq 46 and 48 with eq $78-80$;

$$
\begin{aligned}
& \frac{\tilde{T}^{*}}{m} \ln \left\{\frac{\phi_{0}}{\phi(e)}\right\}-\tilde{T}^{*} \ln \left\{\frac{1-\phi_{0}}{1-\phi(e)}\right\}-\left\{\phi_{0}-\phi(e)\right\} \\
& +\frac{\tilde{T}^{*}}{2 m}\left\{\left(\gamma_{0}-1\right)-\ln \gamma_{0}\right\}+\frac{\tilde{T}^{*}}{2 m}\left(1-\gamma_{0}\right)\left\{\gamma_{0}{ }^{2}+9 \gamma_{0}-4\right. \\
& \left.-\frac{B}{2}\left(1-\gamma_{0}\right)\left(4 \gamma_{0}-3\right)-\frac{B^{2}}{4}\left(1-\gamma_{0}\right)^{2}\right\}=0
\end{aligned}
$$




$$
\begin{gathered}
\frac{2 \phi_{0} \tilde{T}^{*}}{m} \ln \left\{\frac{\phi_{0}}{\phi(e)}\right\}+2 \tilde{T}^{*}\left(1-\phi_{0}\right) \ln \left\{\frac{1-\phi_{0}}{1-\phi(e)}\right\} \\
+2 \tilde{T}^{*}\left(1-m^{-1}\right)\left\{\phi_{0}-\phi(e)\right\}-\left\{\phi_{0}-\phi(e)\right\}^{2} \\
-(m b)^{-1}\left(\gamma_{0}^{-1}-1\right) \phi_{0}^{2}-\frac{\phi_{0} \tilde{T}^{*}}{m}\left[\left\{2-\gamma_{0}\right.\right. \\
\left.\left.-\frac{B}{4}\left(1-\gamma_{0}\right)\right\}\left(1-\gamma_{0}\right)+\ln \gamma_{0}\right]=0
\end{gathered}
$$

where

$$
\tilde{T}^{*}=k T /(2 \omega E)=\tilde{T}\left(1+m^{-1 / 2}\right)^{-2}
$$

From these equations we can obtain $\gamma_{0}$ and $\phi_{0}$, and thus $\dot{\phi}_{0}$ with the aid of eq 78 , as a function of $m, b$, and $\tilde{T}$.

\section{NUMERICAL RESULTS AND DISCUSSIONS}

\section{Behavior Near $T_{\mathrm{c}}$}

The critical exponents for the interfacial tension and the thickness of the interface are $\frac{3}{2}$ and $-\frac{1}{2}$, as shown in eq 68 and 73 , respectively. These values of the critical exponents are generally predicted by a classical theory in which the free energy can be expanded in a Taylor series as in eq $51 .^{13}$

According to eq 69 , in the case of a symmetrical polymer system, $\sigma /\left\{T_{\mathrm{c}}(1-\tilde{T})^{3 / 2}\right\}$ decreases with molecular weight in the $-\frac{1}{2}$ power of $m$, whereas $\sigma /(1-\tilde{T})^{3 / 2}$ increases with molecular weight in the $\frac{1}{2}$ power, i.e., proportional to the unpertubed dimension of a polymer coil. On the other hand, in a polymer solution, both $\sigma /\left\{T_{\mathrm{c}}(1-\tilde{T})^{3 / 2}\right\}$ and $\sigma /(1-\widetilde{T})^{3 / 2}$ decreases with molecular weight in the $-\frac{1}{4}$ power in the range of higher molecular weights (eq 70).

The thickness $L$ of the interface increases with increasing molecular weight in the cases of both a polymer-polymer system and a polymer-solvent system, but in different powers of $m$ as seen in eq 74 and 75 . In a symmetrical polymer system, $L$ is proportional to the unperturbed dimension of a coil, i.e., to the $\frac{1}{2}$ power of $m$, whereas in a polymer solution $L$ is approximately proportional to the $\frac{1}{4}$ power of $m$.

Since $\Delta f^{e}$ appears in $\Delta f$ as a higher order term and is ineffective near $T_{\mathrm{c}}$ as seen in eq 60 and 37 , the change in the chain dimension does not affect $\sigma$ and $L$ near $T_{\mathrm{c}}$ as shown in eq 61 and 73, where $\Delta f \rightarrow \Delta f_{\mathrm{u}}$ and $H \rightarrow h_{\mathrm{c}}$. Therefore, the results obtained near $T_{\mathrm{c}}$ (eq 68 and 73 , or 69 ,
70, 74, and 75) do not depend on whether the chain dimension is variable or not. This may generally be expected without the equation for $f^{e}$ (eq 17), because $f^{\mathrm{e}}$ terms in $f$ (the second term in eq 55) always appear as higher order terms because of the equilibrium condition $\partial f^{e} / \partial \gamma=0$ at $\gamma=1$.

The equations for a polymer solution (an asymmetrical system) near $T_{\mathrm{c}}$ are applicable only within the more narrow range of $T_{\mathrm{c}}-T$ when $m$ is the larger value, because the coefficient of the $\left(\Delta f_{\mathrm{u}}\right)^{2}$ term in eq 60 increases with increasing $m$ and also because the applicability of eq 51 becomes restricted within the more narrow range of $T_{\mathrm{c}}-T$ as $m$ increases.

Eq 69 and 74 for a symmetrical polymer system are rewritten as

$$
\sigma=2\left(k T_{\mathrm{c}} / \omega\right)\left(\lambda^{2}+a^{2}\right)^{1 / 2}(1-\tilde{T})^{3 / 2}
$$

and

$$
L=2\left(\lambda^{2}+a^{2}\right)^{1 / 2}(1-\tilde{T})^{1 / 2}
$$

because of eq 67 . The characteristic interaction length $\left(\lambda^{2}+a^{2}\right)^{1 / 2}$ consists of two terms, the intersegmental interaction length $\lambda$ and the intermolecular interaction length $a$, which is a measure of the chain dimension. When $a^{2}=0$ in eq $69^{\prime}$ and $74^{\prime}$, these equations are reduced to eq 71 and 76 for a regular solution, which accord with the results derived by Cahn and Hilliard. ${ }^{7}$ When $\lambda^{2}$ is neglected compared with $a^{2}$, eq $69^{\prime}$ and $74^{\prime}$ agree with the results of Vrij's theory. ${ }^{6}$ The agreement seems quite reasonable, because in Vrij's theory the interaction length of the intersegmental interactions. was ignored and the chain dimension was assumed to be unaltered, while $f^{\ominus}$ is ineffective near $T_{\mathrm{c}}$ as mentioned above. However, the present theory and Vrij's theory provide quite different results for $\sigma$ and $L$ in a polymer solution, i.e., in an asymmetrical system. In Vrij's theory, the intermolecular correlationlength parameter $l^{\prime}$ was evaluated on the basis of Debye's theory. ${ }^{14}$ Therefore, Vrij's theory predicts that $L$ is proportional to the polymer chain dimension, i.e., to $m^{1 / 2}$, and consequently $\sigma$ does not depend on $m$, i.e., $\sigma \propto m^{0}$ (Appendix III), whereas in the present theory, $\sigma \propto m^{-1 / 4}$ and $L \propto m^{1 / 4}$ in a polymer-solvent system. The reason why the two theories yield different. 
results for an asymmetrical system may be that in the asymmetrical system the equation $c_{1} m_{1} \omega_{1}+c_{2} m_{2} \omega_{2}=1$ (which was used in Vrij's theory) does not hold even when $\gamma=1$, whereas in a symmerical system the equation holds when $\gamma=1$. Namely, asymmetry in molecular size may be an essential cause for the small correlation-length parameter in a polymer solution, as pointed out by Vrij and Esker. ${ }^{11,15}$ According to the recent theory of Vrij and Esker ${ }^{11}$ for the critical opalescence, the length parameter $l^{\prime}$ is

$$
l^{\prime 2}=2\left\langle r_{\mathrm{G}}{ }^{2}\right\rangle
$$

for a polymer-polymer-solvent symmetrical system, and

$$
l^{\prime 2}=2\left\langle r_{\mathrm{G}}{ }^{2}\right\rangle \phi_{\mathrm{c}}
$$

for a polymer-solvent asymmetrical system, where $\left\langle r_{G}{ }^{2}\right\rangle$ is the mean square radius of gyration of the polymer coil, and $\phi_{c}$ is the critical concentration of the polymer. That is, $l^{\prime}$ in a symmetrical polymer system is of the order of chain dimension as Debye's theory predicts, whereas $l^{\prime}$ in a polymer-solvent system is much smaller than Debye's value (because $\phi_{\mathrm{c}} \ll 1$ ). The predictions of eq 89 and 90 were confirmed by the experimental observations of light scattering. ${ }^{15}$ The results for $L$ of the present theory just coincide with these findings. Neglecting $\lambda^{2}$ compared with $a^{2}$, i.e., $b^{2} m \gg 1$, eq 74 or $74^{\prime}$ for a symmetrical system reduces to

$$
L^{2}=(8 / 3)\left\langle r_{\mathrm{G}}{ }^{2}\right\rangle(1-\tilde{T})^{-1}
$$

and eq 75 for an asymmetrical system reduces to

$$
L^{2}=(8 / 3)\left\langle r_{\mathrm{G}}{ }^{2}\right\rangle \phi_{\mathrm{c}}(1-\tilde{T})^{-1}
$$

because of eq 65 and $a^{2}=2\left\langle r_{\mathrm{G}}{ }^{2}\right\rangle / 3$ derived from eq 6 .

The result of the present theory for the dependence of $\sigma$ on $m$ in a polymer solution qualitatively agree with the numerical result of Roe's theory ${ }^{10}$ for $\sigma$ of polystyrene-cyclohexane solutions, where $\sigma$ decreases with increasing $m$ compared at a constant $T_{\mathrm{c}}-T$.

Behavior at $\ddot{\phi}=0$

(1) Symmetrical polymer systems

The values of the expansion factors $\alpha_{0}$ calculated by eq 82 with $b=1$ are shown in Figure 1 as a function of $\tilde{T}$. The values of the

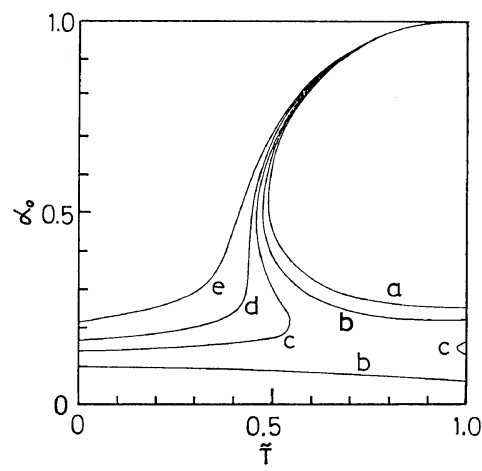

Figure 1. Expansion factor $\alpha_{0}$ as a function of $\tilde{T}^{\text {. }}$ and $m$ for symmetrical system: a, $m=\infty ; b, m=$ 100 ; c, $m=50$; d, $m=34$; e, $m=20$.

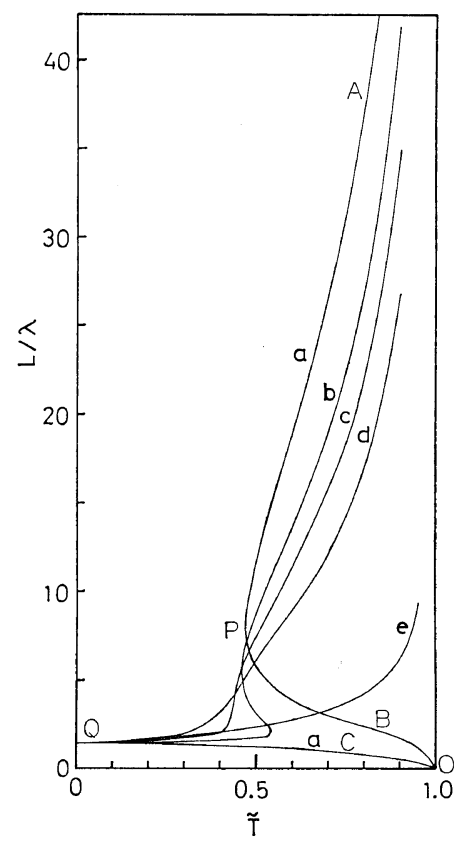

Figure 2. Thickness $L / \lambda$ of interface as a function: of $\tilde{T}$ and $m$ for symmetrical system: a, $m=100$; b, $m=50 ; \mathrm{c}, m=34$; d, $m=20$; e, $m=1$.

interface thickness $L / \lambda$ evaluated from eq 72,78 , and 81 with the values of $\gamma_{0}\left(=\alpha_{0}{ }^{2}\right)$ obtained above are shown in Figure 2. ${ }^{*}$ The thickness of the interface in a lower temperature region

* Even if the higher order terms $\ddot{\phi}$ are taken into account in the derivation of eq. 82 with the aid of eq. 86 , almost the same numerical resulis are obtained for $\sigma$ and $L$. 
does not largely depend on the chain length $m$, and has the limiting value of $L / \lambda=\sqrt{2}$ at $\tilde{T}=0$ independent of $m$ (Appendix IV). $L$ at a low temperature is of the order of a segment length, since the segment-segment interaction length $\lambda$ may be of the order of the segment length. Corresponding to these properties, the expansion factor $\alpha_{0}$ of a chain dimension in the lower temperature region is far from unity, depending on $m$, and is of the order of $m^{-1 / 2}$, which implies that a polymer chain at the interface (at $\dot{\phi}=$ maximum) is almost in a plane or a two dimensional coiled state parallel to the interface.

As shown in Figures 1 and $2, L / \lambda$ and $\alpha_{0}$ have three values for a value of $\tilde{T}$ above about $\tilde{T}=0.5$ for longer molecules, i.e., $m \gtrsim 34$. This suggests an occurence of a transition from a diffuse interface to a sharp interface in polymer systems. The transition may be more clearly seen by considering the behavior of the free energy. The surface free energy $\sigma$ may be semiquantitatively represented by $2(H \Delta f)_{0}{ }^{1 / 2}\left\{\phi^{\prime \prime}(e)-\phi^{\prime}(e)\right\}$ or $2(\Delta f)_{0} L$, because $\sigma$ is given by eq 50 , where $2(H \Delta f)_{0}{ }^{1 / 2}$ and $(\Delta f)_{0}$ are those at $\ddot{\phi}=0$, i.e., at the maximum of the concentration gradient, and are equivalent to their maximum values in the symmetrical system considered here. The calculated values of $2(\Delta f)_{0} L /(E \lambda)$ are shown in Figure 3. For example, comparing $L / \lambda$ with $2(\Delta f)_{0} L /(E \lambda)$ for $m=100$ (curves a in Figures 2

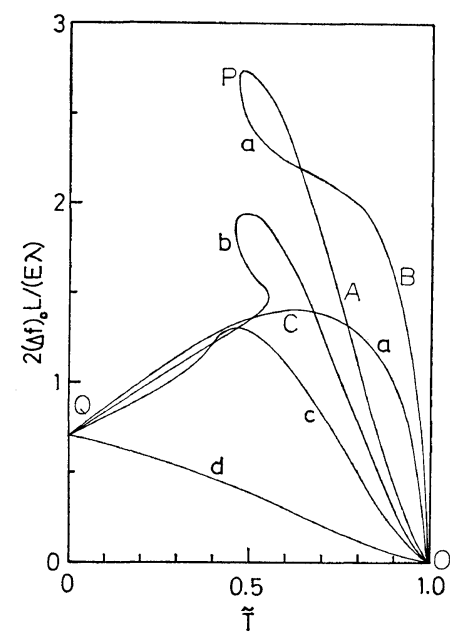

Figure 3. $2(\Delta f)_{0} L /(E \lambda)$, measure of interfacial tension, as a function of $\tilde{T}$ and $m$ for symmetrical systems: a, $m=100 ; \mathrm{b}, m=50$; c, $m=20 ; \mathrm{d}, m=1$. and 3), the curves AP, PBO, and OCQ in Figure 2 correspond to the curves OAP, PBO, and OCQ in Figure 3, respectively. The states represented by the curves OAP and OCQ are stable or metastable, whereas the state designated by the curve PBO is unstable. The free energy curves don't show any typical features of the first order transition, e.g., the curves for $m=100$ have a closed loop in a region of a higher energy part (near a point $P$ ), and the curves for $m=50$ do not have a closed loop or an intersection, which would be due to the insufficient representation of the free energy $\sigma$ by $2(H \Delta f)_{0}{ }^{1 / 2}\left\{\phi^{\prime \prime}(e)-\phi^{\prime}(e)\right\}$. The value of $\sigma$ calculated by intergrating $2(H \Delta f)^{1 / 2}$ with respect to $\phi$ (eq 50) is expected to show the behavior of a typical first order transition (Appendix V). At the transition, the interfacial tension is continuous and its derivative with respect to temperature is discontinuous. The transition predicted is a first order one associated with the change from a diffuse interface to a sharp interface. The qualitative explanation of the transition is as follows. When the temperature decreases from a critical temperature, the thickness of the interface monotonically decreases at first accompanying a mild change in the chain dimension, and as further decreasing of the interface thickness occurs the free energy of $\Delta f_{\mathrm{u}}$ would increase more and more if the chain dimension would not drastically change. Then, at the transition the chain shrinks, sacrificing the increment of the conformational free energy $f^{e}$ so as to make the total free energy $\sigma$ minimum by making the interface thickness smaller and by taking a lower value of $\Delta f_{\mathrm{u}}$. At the transition, the increase of $\Delta f^{e}$ by the chain contraction is balanced with the decrease of $\Delta f_{\mathrm{u}}$ by the decrease in $L$ and $\alpha$.

As seen from Figure 3 the interfacial tension in the lower temperature region below the transition may be weakly dependent on the chain length $m$, having a limiting value of $\sigma / E \lambda=\pi / 4 \sqrt{2}$ independent of $m$ (Appendix IV), whereas $\sigma$ increases with $m$ in the $\frac{1}{2}$ power of $m$ in the vicinity of $T_{\mathrm{c}}$ as already mentioned. These behaviors of $\sigma$ just correspond to those of $L$, i.e., near $T_{\mathrm{c}}$ (above the transition temperature) $L \propto m^{1 / 2}$ and $L(1-\tilde{T})^{1 / 2}$ is of the order 
of the unperturbed chain dimension (eq 74), whereas in a low temperature region $L$ is of the order of the segment length without greatly depending on $m$. These differences in $\sigma, L$, and their $m$ dependences between the high and the low temperature regions suggest the occurrence of a transition from diffuse to sharp interface in high molecular-weight polymer systems.

In most cases of polymer-polymer incompatible systems, the temperature of a usual experimental condition may be much far from $T_{\mathrm{c}}$ (below the transition temperature) because of a low value of mixing entropy. Therefore, the thickness of interface may not be so large, but much less than the unperturbed chain dimension.

The present theory gives a quite different result from Vrij's theory ${ }^{6}$ in the lower temperature region. In Vrij's theory, since the chain dimension was assumed to be invariable, the interfacial tension increased monotonically with decreasing temperature up to $\sigma=(\pi / 4 \sqrt{2}) E a$ at $\tilde{T}=0$, and the interface thickness decreased continuously with decreasing temperature to be $L=\sqrt{2} a$ at $\tilde{T}=0{ }^{*}$ The limiting values of $\sigma$ and $L$ at $\tilde{T}=0$ both increase with increasing $m$, i.e., proportional to the unperturbed chain dimension $a\left(\propto m^{1 / 2}\right)$. However, since the system should be in the lowest energy state at $\tilde{T}=0$, the segment-concentration profile must be a sharp interface, i.e., the system must be completely separated segmentally and not have a diffuse layer, by changing the chain conformation. Therefore, $\sigma$ may tend to a limiting value independent of $m$ at $\tilde{T}=0$. In this sense, the present theory gives more reasonable results than Vrij's theory. The theory of Helfand, et $a l .,{ }^{8,9}$ which may be applicable when the interface thickness $L$ is small, i.e., in the lower temperature region, predicts that $L$ is of the order of $1 \mathrm{~nm}$ in most cases and is much smaller than the unperturbed chain dimension, in qualitative accordance with the result of the present theory in which $L$ at a lower temperature is predicted to be of the order of the segment length, as already mentioned. Roe's

* These results are the same as the equations which are obtained by replacing $\lambda$ by $a$ in the results of the present theory. theory ${ }^{10}$ also gives a similar result for $L$ at a temperature far from $T_{\mathrm{c}}$, i.e., the theory predicts $L$ to be of the order of the lattice separation, which may correspond to the segment length.

(2) Polymer solutions

The values of $\alpha_{0}, \phi_{0}, L / \lambda$, and $2(\Delta f)_{0} L / E \lambda^{*}$

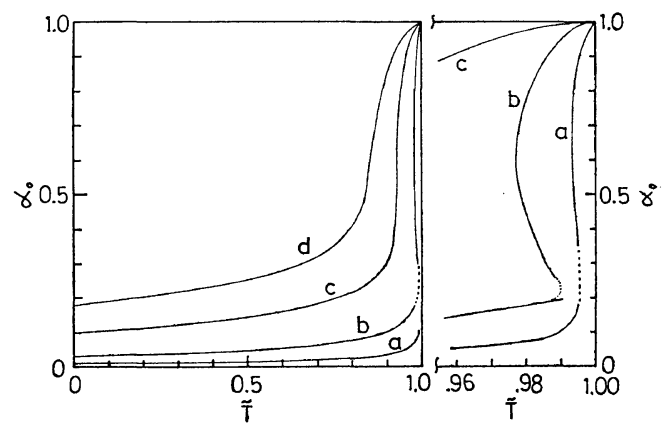

Figure 4. Expansion factor $\alpha_{0}$ as a function of $\tilde{T}^{\text {. }}$ and $m$ for polymer solutions: a, $m=10^{4} ; \mathrm{b}, m=$ $10^{3} ; \mathrm{c}, m=10^{2} ; \mathrm{d}, m=30$. In the regions of the dotted curves connecting the calculated solid curves, reasonable solutions were not obtained probably because the approximation of eq 86 was not sufficient in such regions where solutions of the simultanious equations were very sensitive to. the equations.

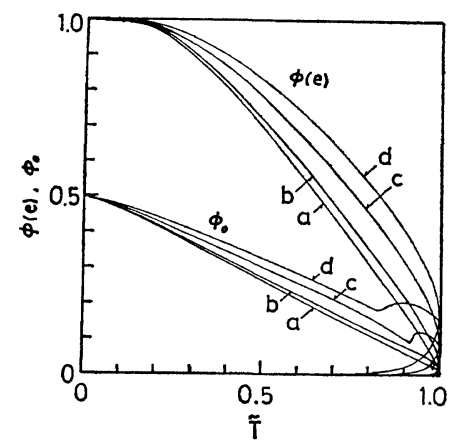

Figure 5. Equilibrium concentration $\phi(e)$ and concentration $\phi_{0}$ at the inflection point of the concentration profile as a function of $\tilde{T}$ and $m$ for polymer solutions. The symbols are the same as those in Figure 4.

* The subscript 0 in $(H \Delta f)_{0}$ and $(\Delta f)_{0}$ indicates: again the value at $\ddot{\phi}=0$ or at the inflection point of concentration profile. Though $(\Delta f)_{0}$ in the present case of a polymer solution does not always. exactly imply the maximum of $\Delta f$ in contrast to a symmetrical case, $(\Delta f)_{0}$ may be very close to the maximum of $\Delta f$. 
$\left[=2(H \Delta f)_{0}{ }^{1 / 2}\left\{\phi^{\prime \prime}(e)-\phi^{\prime}(e)\right\} / E \lambda\right] \quad$ are calculated from the solutions of the simultaneous equations of eq 87 and 88 and shown as a function of $\tilde{T}$ and $m$, in Figures $4,5,6$, and 7, respectively,

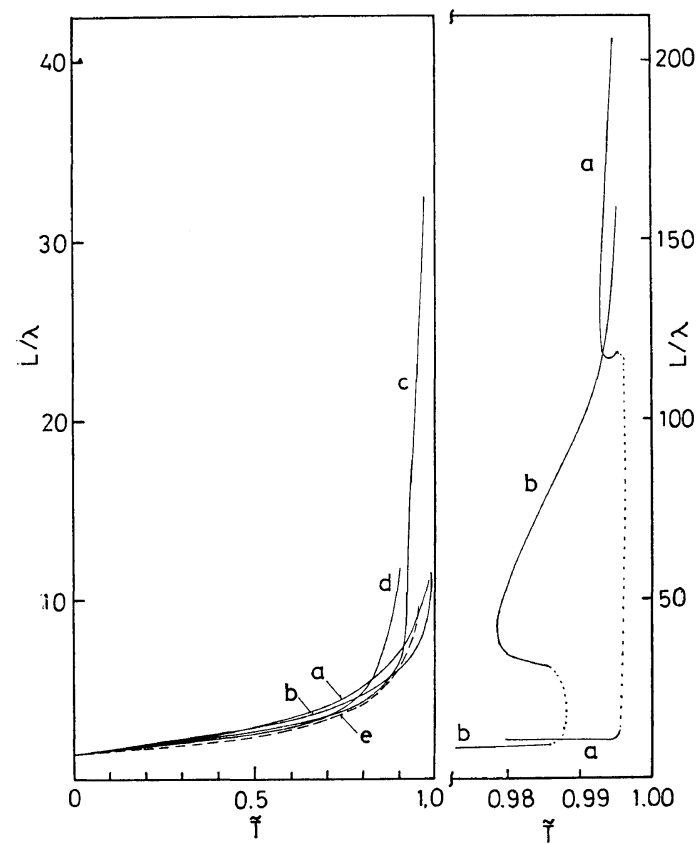

Figure 6. Thickness $L / \lambda$ of interface as a function of $\tilde{T}$ and $m$ for polymer solutions. The symbols are the same as those in Figure 4: e, $m=1$.
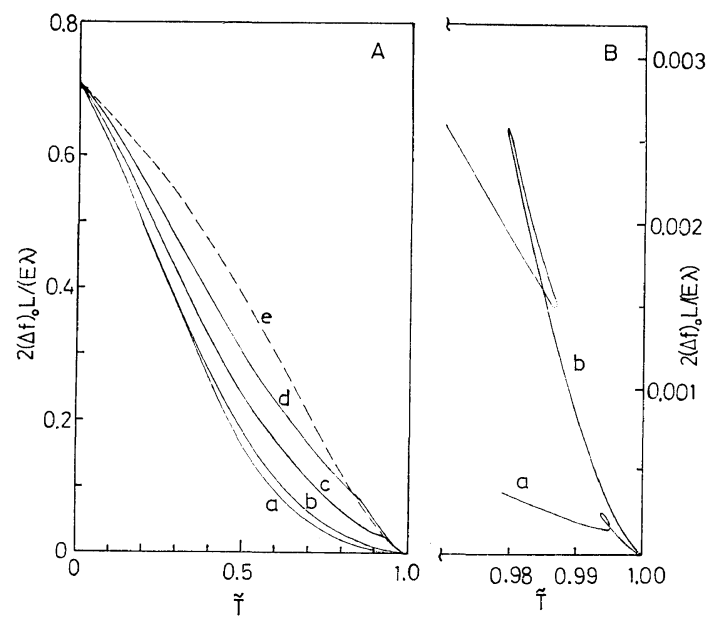

Figure 7. 2( $(\Delta f)_{0} L /(E \lambda)$, measure of interfacial tension, as a function of $\tilde{T}$ and $m$ for polymer solutions. The symbols are the same as those in Figure 4: e, $m=1$. where $b=1$ and $B\left[=8 \phi_{0}{ }^{2} /\left\{\phi^{\prime \prime}(e)-\phi^{\prime}(e)\right\}^{2}\right]$ is taken to be unity because $B$ does not greatly vary except near $T_{\mathrm{c}}$ and does not affect the solutions so much. Although $B$ becomes very large near $T_{\mathrm{c}}$ since $B \propto\left(T_{\mathrm{c}}-T\right)^{-1}$ (from eq 52), the terms containing $B$ in eq 87 and 88 , which appear in the form of $B\left(1-\gamma_{0}\right)$, are ineffective near $T_{\mathrm{c}}$ because the terms containing $B\left(1-\gamma_{0}\right)$ are of the first power of $T_{\mathrm{c}}-T$ near $T_{\mathrm{c}}$ (from eq 52, 59, 72 , and 73).

In a lower temperature region, the dependences of $L / \lambda$ and $\alpha_{0} \mathrm{~m}^{1 / 2}$ on $m$ are very slight, whereas $L / \lambda$ near $T_{\mathrm{c}}$ increases with increasing $m$ in the $\frac{1}{4}$ power of $m$ (eq 75). The limiting value of $L / \lambda$ at $\tilde{T}=0$ is $2^{1 / 2}$ without depending on $m$, and that of $\alpha_{0} m^{1 / 2}$ is $\left(m^{-1}+1\right)^{-1 / 2} \simeq 1$. Therefore the chain at the interface at a low temperature is compressed to be two dimensional just as in a symmetrical system.

The rapid decrease in the thickness of the interface and a drastic compression of a polymer coil at the interface are found as temperature decreases from the critical point. The reduced temperature at which these changes occur becomes higher, and the changes become steeper as $m$ increases. The same transition from a diffuse interface to a sharp interface as in symmetrical systems is also found for polymer solutions of high molecular weight, e.g., for $m=10^{3}$ and $10^{4}$, as shown in Figures 4, 6, and 7 for $\alpha_{0}, L / \lambda$, and $2(\Delta f)_{0} L / E \lambda$. The transition temperature is fairly close to $T_{\mathrm{c}}$ and become closer to $T_{\mathrm{c}}$ as $m$ increases.

The value of $2(\Delta f)_{0} L / E \lambda$, a measure of interfacial tension, decreases slightly with $m$ below the transition temperature, reflecting the change in the phase diagram with $m$, and increases with decreasing temperature to have limiting value of $1 / \sqrt{2}$ without depending on $m$, whereas it may vary with $m$ and $\tilde{T}$, taking the form of $m^{-1 / 4}(1-\tilde{T})^{3 / 2}$ near $T_{\mathrm{c}}$ (eq 70 ).

The concentration at $\ddot{\phi}=0$, i.e., at an inflection point of a $\dot{\phi}-z$ curve, is not a mean value of the concentrations of two equilibrium phases except at temperatures near $T_{\mathrm{c}}$ or near zero, and the concentration profile of a polymer solution is not always symmetrical. The $\phi_{0}$ in Figure 4 suggests that the polymer rich side of the interface layer may be more diffuse than 
the solvent rich side except near $T_{\mathrm{c}}$ and $\tilde{T}=0$.

\section{GENERAL DISCUSSION}

We will discuss here the approximations and model used in the present theory.

\section{Neglect of Higher Order Terms}

In the present treatment, segment concentration $n$ in eq 15 and segment density $\rho$ in eq 5 are expanded in a Taylor's series, neglecting the terms higher than the second order. In general, these approximations are available only for a small gradient, accordingly near $T_{\mathrm{c}}$. Therefore, the reliability of the theory may become worse as the concentration gradient increases with decreasing temperature. As for the expansion of $n$, however, the approximation gives reasonable results even at low temperatures $^{7}$ and may not be so bad, probably because the intersegmental interactions $\left(\varepsilon_{i j}\right)$ are short range ones. On the other hand, the validity of the approximation in the expansion of $\rho$ is not clear. From an optimistic viewpoint, however, the approximation for $\rho$ may also be permissible because the chain dimension of the $z$-direction does not exceed the interface thickness (reciprocal of gradient) even at low temperatures by shrinking of the chain dimension.

Temperature and Concentration Dependence of $E$ $E$, which corresponds to $R T \chi$ in FloryHuggins solution theory, was assumed to be independent of $T$ and $\phi$. But, this is not the case in real systems. The lower critical solution temperature has recently been observed in polymer-polymer mixtures ${ }^{16}$ as well as in polymer solutions, which indicates the existence of a negative entropy term in $E$, i.e., $E$ increases with increasing temperature. Introduction of the temperature dependence of $E$ in the present theory can be easily performed by altering the reduced temperature scale without any change in the formulae of the results obtained. Equation 64 gives the definition of $\tilde{T}$;

$$
\tilde{T}=\frac{k T}{2 \omega E}\left(m_{1}^{-1 / 2}+m_{2}^{-1 / 2}\right)^{2}
$$

where $\tilde{T}$ is not always equal to $T / T_{\mathrm{c}}$ when $E$ depends on $T$. Since a critical temperature is given by putting $\tilde{T}=1$ without depending on the temperature dependence of $E$, we have

$$
\frac{k T_{\mathrm{c}}}{2 \omega E_{\mathrm{c}}}\left(m_{1}^{-1 / 2}+m_{2}^{-1 / 2}\right)^{2}=1
$$

where $E_{\mathrm{c}}$ is $E$ at $T=T_{\mathrm{c}}$. Defining a new temperature scale $\tilde{T}^{\prime}$ as $\tilde{T}^{\prime}=T / T_{\mathrm{c}}, \tilde{T}^{\prime}$ is written in terms of the old scale $\tilde{T}$ by using eq 93 and 94 if $E$ is given as a function of temperature $T$. By the scale transformation $\tilde{T} \rightarrow \tilde{T}^{\prime}$ with the relation thus obtained any results obtained in the present paper are available even when $E$ has entropy terms, since the temperature independence of $E$ was not assumed in the derivations of any basic equations. ${ }^{*}$ When $E$ is expressed by a linear function of $T$, i.e.,

$$
E=e-s T \text {, }
$$
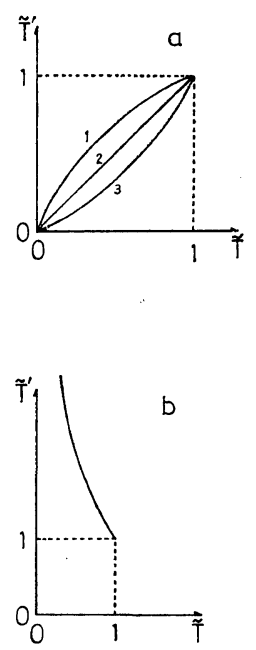

Figure 8. Transformation of temperature scale: a, $\operatorname{UCST}(e>0,2 k+m \omega s>0)(1, s<0 ; 2, s=0 ; 3$, $s>0) ; \mathrm{b}, \operatorname{LCST}(e<0,2 k+m \omega s<0)$.

* The temperature independence of $E$ was used in evaluation of $\beta$ (eq 62) which was defined by eq 53. However, $\tilde{T}$ in the expressions for the properties near $T_{\mathrm{c}}$ was not defined by eq 93, but as a temperature reduced by a true critscal temperature. Consideration of the temperature dependence of $E$ in evaluation of $\beta$ implies the changing of the temperature scale. Therefore, by changing the temperature scale in the expressions of $\sigma$ and $L$ near $T_{\mathrm{c}}$ in the same way as the others, these expressions also valid even when $E$ has some temperature dependenco. 
a new temperature scale, $\tilde{T}^{\prime}=T / T_{\mathrm{c}}$, is written in terms of the old scale $\tilde{T}$ as

$$
\begin{aligned}
\tilde{T}^{\prime}=\tilde{T} & \left\{k+2\left(m_{1}{ }^{-1 / 2}+m_{2}{ }^{-1 / 2}\right)^{-2} \omega s\right\} \\
& \times\left\{k+2\left(m_{1}{ }^{-1 / 2}+m_{2}{ }^{-1 / 2}\right)^{-2} \omega s \tilde{T}\right\}^{-1}
\end{aligned}
$$

using eq 93, 94, and 95. From eq 96 we have $\left(1-\tilde{T}^{\prime}\right)=(1-\tilde{T}) k\left\{k+2\left(m_{1}^{-1 / 2}+m_{2}{ }^{-1 / 2}\right) \omega s\right\}^{-1}$ near $T_{\mathrm{c}}$. Figure 8 schematically shows the scale transformations for two cases, i.e., systems having a lower and an upper critical solution temperatures.

On the other hand, the concentration dependence of $E$ alters the results obtained, and it is not easy from the present results quantitatively to estimate the properties for the case in which $E$ depends on $\phi$. However, if the dependency of $E$ on $\phi$ is mild, the qualitative properties may not be changed.

In the present model for the free energy $\vec{F}$, which is essentially based on Flory-Huggins solution theory, the random mixing, i.e., the uniformity of the segment concentration in the system is assumed in an equillibrium phase in considering the local free energy and the segment density of a chain, which may be permissible only in a concentrated solution in a polymer system. Hence, in a system including a very diluted phase the present approximation may not be sufficient for evaluation of the free energy. Although as for the free energy $\Delta f_{\mathrm{u}}$ the deviation from the random mixing may formally be accounted for by introducing the concentration dependence of $E$, the excluded volume effect associated with intramolecular segment-segment interactions must also be accounted for in a dilute solution system. The chain dimension may be much less than the unperturbed one below $T_{\mathrm{c}}$ even in a bulk (but dilute) phase because $T_{\mathrm{c}}$ is lower than the $\Theta$ temperature.

Segment Density $\rho$ and Conformational Free Energy $f^{\mathrm{e}}$

Equation 17, which expresses the conformational entropy for a chain having a segment density given by eq 6 , does not have any theoretical provision for non-Gaussian or nonuniform expansion as in the present case, as already mentioned. Apart from this problem of non-uniform expansion, the excluded volume effect is not taken into account in eq 17 , which is important especially when a polymer coil is much compressed. According to eq 17 or 39, $f^{e}$ increases with decreasing $\alpha$ only logarithmically, and $\alpha$ can take a value much less than $m^{-1 / 2}$, which means the compression of segment length, without having so high a value of the free energy. But, actually, $f^{\mathrm{e}}$ must increase more rapidly as $\alpha$ approaches to $m^{-1 / 2}$ because of the excluded volume effect due to self-avoiding or the hard core of the segment and the short range (or intrasegmental) interactions. Therefore, an actual thickness of the interface at low temperatures may be larger than the thickness obtained in the present theory. The excluded volume effect may also induce some chain expansion to the $x$ - and $y$-directions accompanying the compression of the $z$-direction, which may, however, give no influence to the intermolecular interaction free energy $f_{\mathrm{u}}$ because there are no gradients in the $x$ - and $y$-directions.

As mentioned above, there remain many problems to be solved. But, the author believes that the possibility of the transition or the drastic change in the interface thickness is reasonably predicted if the combinatorial mixing entropy and the magnitude of elastic free energy of a chain used here are available, although the quantitative results are much affected by the model and approximations adopted. Roe's theory, ${ }^{10}$ however, does not give such a transition, although the distortion of polymer molecules was also taken into account there. This inconsistency probably comes from the difference in evaluating the effect of conformational entropy at the interface on the local free energy, which closely relates to the combinatorial entropy of mixing. This is also associated with the problem of how to recognize polymer molecules as molecules in a non-uniform concentrated solution.

The transition predicted here seems similar to the globule-coil transition which has been recently suggested, but not proved, to occur in an isolated chain molecule below the $\Theta$ temperature. ${ }^{17}$ However, in the present transition the force compressing a coiled chain is an anisotropic and one-dimensional force acting so as to reduce the interface excess free energy, whereas in the latter transition the force may be an isotropic and three-dimensional one due 
to the intramolecular excluded volume of negative sign, if these transitions occur.

Finally it is noted that the present treatment may apply to other phenomena of non-uniform polymer systems, e.g., to the spinodal decomposition in polymer systems, where fluctuations of both segment and molecular center compositions may in general have to be considered.

\section{APPENDIX}

\section{Approximation of eq 23}

Since an approximation of $\bar{\alpha}$ for evaluation of the exponential term in eq 22 may suffice if it is available in the range of $r_{z}^{2} /\left(a^{2} \bar{\alpha}^{2}\right)<1, r_{z} / \bar{\alpha}$ defined by eq 10 may be written in the expansion form as

$$
\begin{aligned}
r_{z} / \bar{\alpha}= & \int_{0}^{-r_{z}} \mathrm{~d} r_{z}{ }^{\prime}\left\{\alpha(z)^{-1}+\left(\dot{\alpha}^{-1}\right)\left(r_{z}{ }^{\prime}+r_{z}\right)\right. \\
& \left.+\left(\ddot{\alpha}^{-1}\right)\left(r_{z}{ }^{\prime}+r_{z}\right)^{2} / 2+\cdots\right\} \\
= & -r_{z} / \alpha+\left(r_{z} / \alpha\right)^{2} \dot{\alpha} / 2+\left(r_{z} / \alpha\right)^{3}\left(\alpha \ddot{\alpha}-2 \dot{\alpha}^{2}\right) / 6+\cdots
\end{aligned}
$$

Here we will use the following approximation for $x$ satisfying $a^{2} x^{2}<1$

$$
\begin{aligned}
& \exp \left(-a^{2} x^{2}-b x^{3}-c x^{4}\right) \\
& \quad=\left\{\exp \left(-a^{2} x^{2}\right)\right\}\left\{\exp \left(-b x^{3}-c x^{4}\right)\right\} \\
& \quad \simeq\left\{\exp \left(-a^{2} x^{2}\right)\right\}\left(1+b x^{3}-c x^{4}-\frac{b^{2}}{2} x^{6}+\cdots\right)
\end{aligned}
$$

where $\left|b x^{3}\right|$, and $\left|c x^{4}\right| \ll 1$ for $a^{2} x^{2}<1$. Then, using eq A-1, we have

$$
\begin{aligned}
& \exp \left(-\frac{r_{z}^{2}}{a^{2} \bar{\alpha}^{2}}\right) \simeq\left\{\exp \left(-\frac{r_{z}^{2}}{a^{2} \alpha^{2}}\right)\right\} \\
& \quad \times\left[1+\frac{\dot{\alpha}}{a^{2} \alpha^{3}} r_{z}{ }^{3}-\left\{\frac{\dot{\alpha}^{2}}{4 \alpha}-\frac{\ddot{\alpha}}{3}\right\}\left(\alpha^{3} a^{2}\right)^{-1} r_{z}{ }^{4}+\frac{\dot{\alpha}^{2}}{2 \alpha^{6} a^{2}} r_{z}^{6}\right]
\end{aligned}
$$

which is eq 23 .

II. Approximation of eq 86

The concentration profile near $T_{\mathrm{c}}$ is expressed by $^{13}$

$$
\begin{aligned}
\phi= & \frac{1}{2}\left\{\phi^{\prime \prime}(e)+\phi^{\prime}(e)\right\} \\
& +\frac{1}{2}\left\{\phi^{\prime \prime}(e)-\phi^{\prime}(e)\right\} \tanh \left\{2\left(z-z_{0}\right) / L\right\}
\end{aligned}
$$

in the classical theory, where $z_{0}$ is $z$ at $\ddot{\phi}=0$, and $L$ is the thickness of interface defined by eq 72. Assuming that the concentration profile just near the position of $\ddot{\phi}=0$, i.e., near $z_{0}$, is expressed by the form of eq A-4 in a whole temperature region even in an asymmetrical system, differentiation of eq A-4 gives the relation

$$
\dddot{\phi}\left(z_{0}\right)=-\frac{8}{L^{2}} \dot{\phi}\left(z_{0}\right)
$$

which is equivalent to eq 86 because of eq 72 . III. $\sigma$ and $L$ near $T_{\mathrm{c}}$ based on Vrij's theory

According to Vrij's theory, ${ }^{6} h_{\mathrm{c}}$ in eq 61 and 73, which corresponds to $\Omega l^{2} / 12$ in Vrij's theory (see eq 34 in ref 6), is given by

$$
h_{\mathrm{c}}=\frac{1}{12}\left(\frac{W_{11}^{\prime}}{\omega_{1}^{\prime 2}} l_{11}^{\prime 2}-\frac{2 W_{12}^{\prime}}{\omega_{1}^{\prime} \omega_{2}{ }^{\prime}} l_{12}^{\prime 2}+\frac{W_{22}^{\prime}}{\omega_{2}^{\prime 2}} l_{22}^{\prime 2}\right)
$$

where $W^{\prime}, l^{\prime}$, and $\omega^{\prime}$ are for a molecule, whereas in the present theory they $(W, l$, and $\omega)$ are for a segment. When the segment density of a polymer coil is assumed to be Gaussian, we obtain $h_{\mathrm{c}}$ from eq A-6 as

$$
h_{\mathrm{c}}=\frac{a^{2}}{4}\left(\frac{W_{11}^{\prime}}{\omega_{1}^{\prime 2}}-\frac{W_{12}^{\prime}}{\omega_{1}^{\prime} \omega_{2}^{\prime}}\right) \equiv \frac{a^{2}}{4} \bar{e}
$$

disregarding the segment interaction length as compared with the chain dimension. Then, from eq 61 and 73 with eq A-7, we derive $\sigma$ and $L$ for a polymer solution based on Vrij's theory as

$$
\sigma_{\left(T \sim T_{\mathrm{C}}\right)}=2\left(a / m^{1 / 2}\right)(\bar{e} / 2 \bar{\Omega})^{1 / 2}(1-\tilde{T})^{3 / 2}
$$

and

$$
L_{\left(T \sim T_{\mathrm{c}}\right)}=2 a(\bar{e} / 2 \bar{\Omega})^{1 / 2}(1-\tilde{T})^{-1 / 2}
$$

IV. $\sigma$ and $L$ at $\tilde{T}=0$.

From eq 40, we obtain

$$
m_{1} \omega_{1} E+k T \ln \phi_{1}{ }^{\prime}(e)=0
$$

when $T$ is near to zero. Substituting eq A-10 into eq 38 and putting $\tilde{T}=0$, i.e., $T=0$, yields

$$
\Delta f(\tilde{T}=0)=E\left(\phi-\phi^{2}\right)
$$

Equations 31, 32, and 49 (with eq 66) give

$$
H(\tilde{T}=0)=\bar{\Omega}=E \lambda^{2} / 2
$$

Substituting eq A-11 and A-12 into eq 50, we can derive

$$
\sigma=2 \int_{0}^{1}\left\{\bar{\Omega} E\left(\phi-\phi^{2}\right)\right\} \mathrm{d} \phi=\pi E \lambda / 4 \sqrt{2}
$$


On the other hand, using eq A-11, A-12, and $48, L$ defined by eq 72 is expressed by

$$
L_{(\tilde{T}=0)}=\sqrt{2} \lambda
$$

noticing that $\phi_{0}=\frac{1}{2}$ at $\tilde{T}=0$.

V. Calculation of the Value of $2(H \Delta f)^{1 / 2}$ Near $\phi=\frac{1}{2}$ in a symmetrical System

The value of $2(H \Delta f)^{1 / 2}$ near $\ddot{\phi}=0$ can be calculated as a function of $\phi$ using a method which is schematically shown in Figure A-1.

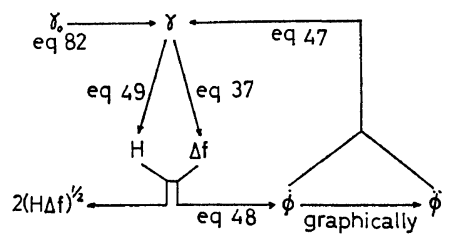

Figure A-1. Method of calculation of $2(H \Delta f)^{1 / 2}$ as a function of $\phi$ near $\ddot{\phi}=0$.

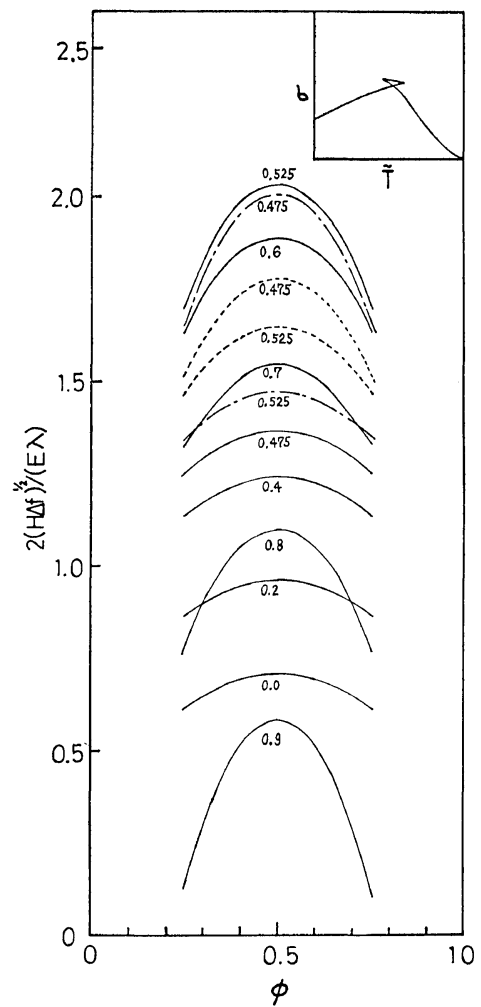

Figure A-2. $2(H \Delta f)^{1 / 2} /(E \lambda)$ as a function of $\phi$ at various $\widetilde{T}$ for a symmetrical system of $m=50$. The numbers in the figure indicate the values of $\tilde{T}$.
First, using the value of $\gamma_{0}$ in place of $\gamma$, which is calculated by eq 82 , we compute the approximate values of $H$ and $\Delta f$ near $\phi=\frac{1}{2}$ as a function of $\phi$ with the use of eq 49 (with eq 31 and 79 of $\ddot{\phi}=0$ ) and eq 37 (with eq 38 and 39), respectively. Then, $\dot{\phi}$ is evaluated as a function $\phi$ from eq 48 with the values of $H$ and $\Delta f$ thus obtained and $\ddot{\phi}$ is also given by graphically differentiating the $\dot{\phi} v s$. $\phi$ curves just obtained. From those values of $\dot{\phi}$ and $\ddot{\phi}$, the value of $\gamma$ is calculated with eq 47 as a function of $\phi$. Next, using this value of $\gamma$, the values of $H$ and $\Delta f$ are again calculated by eq 37 and 49 . Repeating this process, the obtained values of $\dot{\phi}, \ddot{\phi}$, and $\gamma$ are converged. Thus, the values of $H$ and $\Delta f$, therefore $2(H \Delta f)^{1 / 2}$, are obtained as a function of $\phi$. The $2(H \Delta f)^{1 / 2} / E \lambda v s$. $\phi$ curves near $\phi=\frac{1}{2}$ obtained, for example, for $m=50$ are shown in Figure A-2. As seen from the figure, $\sigma$ calculated by integrating $2(H \Delta f)^{1 / 2}$ with respect to $\phi$ (eq 50) is expected to show the typical behavior of the first order transition, as schematically shown at the top of the figure.

\section{REFERENCES}

1. G. Langhammer and L. Nester, Macromol. Chem., 88, 179 (1965).

2. N. A. Krotova and L. P. Morogova, Kolloid Zh., 26, 207 (1964).

3. V. K. Gromov and A. E. Chalykh, Vysokomol. Soedin., 7, 220 (1965).

4. S. S. Voyutskii, A. N. Kamenskii, and N. M. Fodiman, Kolloid. Z.-Z. Polymere, 215, 36 (1967); Vysokomol. Soedin., 11, 394 (1969).

5. J. Letz, J. Polym. Sci., A-2, 7, 1987 (1969).

6. A. Vrij, ibid., A-2, 6, 1919 (1968).

7. J. W. Cahn and J. E. Hilliard, J. Chem. Phys., 28, 258 (1958).

8. E. Helfand and Y. Tagami, J. Polym. Sci., B-9, 741 (1971); J. Chem. Phys., 56, 3592 (1972); ibid., 57, 1812 (1972).

9. E. Helfand and A. M. Sapse, J. Chem. Phys., 62, 1327 (1975).

10. R. J. Roe, ibid., 62, 490 (1975).

11. A. Vrij and M. W. J. van den Esker, J. Chem. Soc. Faraday Trans. II, 68, 513 (1972).

12. e.g., P. J. Flory, "Principles of Polymer Chemistry," Cornell University Press, Ithaca, N.Y., 1953.

13. J. Pressing and J. E. Mayer, J. Chem. Phys., 
Theory of Liquid-Liquid Interface of Polymer Systems

59, 2711 (1973).

14. P. Debye, ibid., 31, 680 (1959). 15. A. Vrij, J. Polym. Sci., C44, 169 (1974); A.
Vrij and M. W. J. van den Esker, J. Polym. Sci., Phys. Ed., 13, 727 (1975).

16. M. Bank, J. Leffingwell, and C. Thies, ibid.,
A-2, 1097 (1972); L. P. McMaster, Macromolecules, 6, 760 (1973).

17. O. B. Ptitsyn, A. K. Kron, and Yu. Ye. Eizner, J. Polym. Sci., C16 3509 (1968); Yu. Ye. Eizner, Polymer Sci., USSR, 11, 409 (1969); C. Domb, Polymer, 15, 259 (1974). 